\title{
Graded Modalities in Strategy Logic ${ }^{\text {药 }}$
}

\author{
Benjamin Aminof ${ }^{\mathrm{a}}$, Vadim Malvone ${ }^{\mathrm{b}}$, Aniello Murano ${ }^{\mathrm{b}}$, Sasha Rubin ${ }^{\mathrm{b}}$ \\ ${ }^{a}$ Technische Universitat Wien, Austria \\ ${ }^{b}$ Università degli Studi di Napoli Federico II, Italy
}

\begin{abstract}
Strategy Logic (SL) is a logical formalism for strategic reasoning in multi-agent systems. Its main feature is that it has variables for strategies that are associated to specific agents using a binding operator. In this paper we introduce Graded Strategy Logic (GRADEDSL), an extension of SL by graded quantifiers over tuples of strategy variables, i.e., "there exist at least $g$ different tuples $\left(x_{1}, \ldots, x_{n}\right)$ of strategies" where $g$ is a cardinal from the set $\mathbb{N} \cup\left\{\aleph_{0}, \aleph_{1}, 2^{\aleph_{0}}\right\}$. We prove that the model-checking problem of GRADEDSL is decidable. We then turn to the complexity of fragments of GRADEDSL. When the $g$ 's are restricted to finite cardinals, written GRADED $\mathrm{SL}$, the complexity of model-checking is no harder than for SL, i.e., it is non-elementary in the quantifier-block rank. We illustrate our formalism by showing how to count the number of different strategy profiles that are Nash equilibria (NE). By analysing the structure of the specific formulas involved, we conclude that the important problem of checking for the existence of a unique NE can be solved in 2ExPTIME, which is not harder than merely checking for the existence of such an equilibrium.
\end{abstract}

Keywords: Strategic logics; Graded modalities; Nash equilibria.

\section{Introduction}

Strategy Logic (SL) is a powerful formalism for reasoning about strategies in multi-agent systems [3, 4. Strategies tell an agent what to do - they are functions that prescribe an action based on the history. The key idea in SL is to treat strategies as first-order objects. A strategy $x$ can be quantified existentially $\langle\langle x\rangle\rangle$ (read: there exists a strategy $x$ ) and universally $\llbracket[x \rrbracket$ (read: for all strategies $x$ ). Furthermore, strategies are not intrinsically glued to specific agents: the binding operator $(\alpha, x)$ allows one to bind an agent $\alpha$ to the strategy $x$. SL strictly subsumes several other logics for strategic reasoning including the well known ATL and ATL ${ }^{\star}[5$. Being a very powerful logic, SL can directly express many solution concepts [6, 4, 7, 8, 9, 10, 11, 12, among which that a

\footnotetext{
This is an extended version of the works [1, 2].
} 
strategy profile $\bar{x}$ is a Nash equilibrium, and thus also the existence of a Nash equilibrium (NE).

The Nash equilibrium is one of the most important concepts in game theory, forming the basis of much of the recent fundamental work in multi-agent decision making. A challenging and important aspect is to establish whether a game admits a unique NE 13, 14, 15. This problem is relevant to the predictive power of NE since, in case there are multiple equilibria, the outcome of the game cannot be uniquely pinned down [16, 17, 18. Unfortunately, uniqueness has mainly been established either for special cost functions [13], or for very restrictive game topologies [19]. Moreover, there is no general theory of when games have unique equilibria that can be applied to different application areas [13.

In this paper, we address and solve the problem of expressing the uniqueness of certain solution concepts (and NE in particular) in a principled and elegant way, by introducing an extension of SL called GradedSL. More specifically, we extend SL by replacing the quantification $\langle\langle x\rangle\rangle$ and $\llbracket x \rrbracket$ over strategy variables with graded quantification over tuples of strategy variables: $\left\langle\left\langle x_{1}, \ldots, x_{n}\right\rangle\right\rangle^{\geq g}$ $\left(\right.$ read $\left\langle\left\langle x_{1}, \ldots, x_{n}\right\rangle\right\rangle^{\geq g}$ as "there exist at least $g$ different tuples $\left(x_{1}, \ldots, x_{n}\right)$ of strategies") and its dual $\llbracket\left[x_{1}, \ldots, x_{n} \rrbracket\right]^{<g}$, where $g \in \mathbb{N} \cup\left\{\aleph_{0}, \aleph_{1}, 2^{\aleph_{0}}\right\}$. Here, two tuples are different if they are different in some component, and two strategies are different if they disagree on some history. The key is being able to express uniqueness of $\mathrm{NE}$ is the combination of quantifying over tuples (instead of singleton variables), and adding counting (in the form of graded modalities).

As far as the expressive power of GRADEDSL concerns, we prove that counting strategies in SL is not possible in general (see Theorem 2.1). On the other hand, every formula of SL has an equivalent formula of GRADEDSL formed by replacing every quantifier $\langle\langle x\rangle\rangle$ with $\langle\langle x\rangle\rangle^{\geq 1}$. Additionally, the possibility of quantifying over tuples of strategy variables (rather than single strategies) makes the logic quite expressive.

We address the model-checking problem for GRADEDSL and prove that it is decidable. We also address the complexity of several fragments of GRADEDSL. First we consider the case in which the $g$ 's are restricted to finite cardinals, written GRADED $\mathrm{N}$ SL. Then we investigate the graded extension of classic fragments of SL, such as Nested-Goal SL and one-goal SL 4, while maintaining the restriction of grades over finite cardinals. Roughly speaking, the Nested-Goal restriction encompasses formulas in a special prenex normal form with a particular nested temporal structure that restricts the application of both strategy quantifiers and agent bindings; further, the one-goal restriction is obtained by forbidding any nesting and Boolean operation over bindings (see Section 2.4 for details).

We show that the complexity of the model-checking problem for GRADED $\mathbb{N} S L$ is no harder than for SL, i.e., it is non-elementary in the nesting depth of quantifiers. In particular, we show that model checking GRADED $\mathrm{NL}_{\mathbb{N}}$ formulas with a nesting depth $k>0$ of blocks of quantifiers (a block of quantifiers is a maximally-consecutive sequence of quantifiers of the same type, i.e., either all existential, or all universal) is in $(k+1)$ ExPTIME, and that for the special 
case where the formula starts with a block of quantifiers, it is in $k$ ExPTimE. Since many natural formulas contain a very small number of quantifiers, the complexity of the model-checking problem is not as bad as it seems. Specifically, several solution concepts can be expressed as SL formulas with a small number of quantifiers [6, 4, 7, 8, 9]. Since the existence of a NE, and the fact that there is at most one NE, can be expressed in GRADED $\mathrm{NL}$ using simple formulas (assuming that the agents' goals are given as LTL formulas) we are able to conclude that the problem of checking the uniqueness of a NE can be solved in 2ExPTIME. Previously, it was known that existence of NE can be checked in 2ExPTIme [4, 7]. Thus, GRADEDSL is the first logic that can solve the existence and uniqueness of $\mathrm{NE}$ (as well as many other solution concepts) in 2ExpTIME.

Concerning the graded Nested-Goal fragment, namely GRADEDSL[NG], we show that, in case the $g$ 's are restricted to finite cardinals, it has the same modelchecking complexity as Nested-Goal SL, i.e., non-elementary in the alternation number of the quantifiers appearing in the formula (the alternation number is, roughly speaking, the maximum number of existential/universal quantifier switches [4]). For the one-goal fragment, namely GraDED $\mathrm{SL}_{\mathbb{N}}[\mathrm{G}]$, the model checking problem is instead 2ExPTIME-COMPLETE. All model checking complexities reported so far refer to the size of the formula. Instead, with respect to the size of the model, the model-checking problem is PTIME-COMPLETE for all these cases.

Related work. The importance of solution concepts, verifying a unique equilibrium, and the relationship with logics for strategic reasoning is discussed above. We now give some highlights from the long and active investigation of graded modalities in the formal verification community.

Graded modalities were first studied in modal logic [20] and then exported to the field of knowledge representation to allow quantitative bounds on the set of individuals satisfying a given property. Specifically, they were considered as counting quantifiers in first-order logics [21] and number restrictions in description logics [22]. Graded $\mu$-calculus, in which immediate-successor accessible worlds are counted, was introduced to reason about graded modal logic with fixed-point operators [23. Recently, the notion of graded modalities was extended to count the number of paths in the branching-time temporal logic formulas CTL and CTL ${ }^{\star}$ 24, 25]. In the verification of reactive systems, we mention two orthogonal approaches: module checking ${ }^{1}$ for graded $\mu$-calculus 28,29 and an extension of ATL by graded path modalities [30.

The work closest to ours is [31]: also motivated by counting NE, it introduces a graded extension of SL, called GSL. In contrast with our work, GSL has a very intricate way of counting strategies: it gives a semantic definition for when

\footnotetext{
${ }^{1}$ Module checking is a decision problem proposed in late 1990 s to formalize verification of open systems, i.e., systems that must adapt their behavior to the input they receive from the environment [26]. Recently it has been showed that module checking offers a distinctly different perspective from the problem of model checking [27].
} 
two strategies should be considered equal, and counts the number of equivalence classes. While this approach is justified, it leads to a complicated model-checking problem. Indeed, only a very weak fragment of GSL has been solved in [31] by exploiting an ad hoc solution that does not seem to be easily scalable to (all of) GSL. Precisely, the fragment investigated there is the vanilla restriction of the graded version of one-goal SL 32. There is a common belief that the one-goal fragment is not powerful enough to express the existence of a Nash Equilibrium in concurrent games. The smallest fragment that is known to be able to represent this is the so called Boolean-goal Strategy Logic, whose graded extension (in the GSL sense) has no known solution ${ }^{2}$

Outline. The sequel of the paper is structured as follows. In Section 2 we introduce GRADEDSL and provide some preliminary related concepts. In Section 3 we address the model-checking problem for GRADEDSL and its fragments. In Section 4 we illustrate our logic by expressing the uniqueness of various solution concepts. We conclude with Section 5 in which we have a discussion and suggestions for future work.

\section{Graded Strategy Logic}

In this section we introduce Graded Strategy Logic, which we call GRADEDSL for short.

In the following we use a finite set of variables $\mathrm{Vr}$, a finite set of agents $\mathrm{Ag}$, and a finite set of atomic propositions AP. We denote variables by $x_{i}, x_{j}$, etc., agents by $\alpha_{i}, \alpha_{j}$, etc., and atomic propositions by $p$, etc. The assumption that these sets are finite is simply a technical convenience: the model-checking problem (Definition 3.1) takes as input formulas and arenas with any number of variables, agents, and atoms.

\subsection{Syntax}

GRADEDSL extends SL by replacing the singleton strategy quantifiers $\langle\langle x\rangle\rangle$ and $[[x]]$ with the graded (tupled) quantifiers $\left\langle\left\langle x_{1}, \ldots, x_{n}\right\rangle\right\rangle^{\geq g}$ and $\left[\left[x_{1}, \ldots, x_{n}\right]^{<g}\right.$, respectively, where $g \in \mathbb{N} \cup\left\{\aleph_{0}, \aleph_{1}, 2^{\aleph_{0}}\right\}$ is called the grade of the quantifier. Intuitively, these are read as "there exist at least $g$ tuples of strategies $\left(x_{1}, \ldots, x_{n}\right)$ " and "all but less than $g$ many tuples of strategies", respectively. The syntax $(\alpha, x)$ denotes a binding of the agent $\alpha$ to the strategy $x$.

Definition 2.1. GRADEDSL formulas are built inductively by means of the following grammar, where $p \in \mathrm{AP}, \alpha \in \mathrm{Ag}, x, x_{1}, \ldots, x_{n} \in \operatorname{Vr}$ such that $x_{i} \neq x_{j}$ for $i \neq j$ and $n \in \mathbb{N}$, and $g \in \mathbb{N} \cup\left\{\aleph_{0}, \aleph_{1}, 2^{\aleph_{0}}\right\}$ :

\footnotetext{
${ }^{2}$ In 33 it has been shown that, in the restricted case of turn-based structures it is possible to express the existence of Nash equilibria in $m^{-} \mathrm{ATL}^{\star}$ [34, a memory-full variant of $\mathrm{ATL}^{\star}$ (hence included in one-goal SL), but exponentially more succinct — and thus with a much more expensive model-checking algorithm. As also the authors in [33] state, it is not clear how to extend this result to the concurrent setting, even in the two player case.
} 


$$
\varphi:=p|\neg \varphi| \varphi \vee \varphi|\mathrm{X} \varphi| \varphi \mathrm{U} \varphi\left|\left\langle\left\langle x_{1}, \ldots, x_{n}\right\rangle\right\rangle^{\geq g} \varphi\right|(\alpha, x) \varphi .
$$

Note that GradeDSL formulas are defined w.r.t. fixed finite sets of atomic propositions AP, agents Ag, and variables $\mathrm{Vr}$.

Notation. Whenever we write $\left\langle\left\langle x_{1}, \ldots, x_{n}\right\rangle\right\rangle^{\geq g}$ we mean that $x_{i} \neq x_{j}$ for $i \neq j$, i.e., the variables in a tuple are distinct (note that this does not mean that the strategies the variables represent are distinct).

Shorthands are derived as usual. Specifically, true $\triangleq p \vee \neg p$, false $\triangleq \neg$ true, $\mathrm{F} \varphi \triangleq \operatorname{true} \mathrm{U} \varphi$, and $\mathrm{G} \varphi \triangleq \neg \mathrm{F} \neg \varphi$. Also, we have that $\left[\left[x_{1}, \ldots, x_{n}\right]\right]^{<g} \varphi \triangleq$ $\neg\left\langle\left\langle x_{1}, \ldots, x_{n}\right\rangle\right\rangle^{\geq g} \neg \varphi$. The operators $\left\langle\left\langle x_{1}, \ldots, x_{n}\right\rangle\right\rangle^{\geq g}$ (resp. $\left[\left[x_{1}, \ldots, x_{n}\right]\right]^{<g}$ ) are called existential (resp. universal) strategy quantifiers.

In order to define the semantics, we first define the concept of free placeholders in a formula, which refer to agents and variables. Intuitively, an agent or variable is free in $\varphi$ if it does not have a strategy associated with it (either by quantification or binding) but one is required in order to ascertain if $\varphi$ is true or not. The definition mimics that for SL 4. It is important for defining the model-checking procedure, in particular for the encoding of strategies as trees (Definition 3.3).

Definition 2.2. The set of free agents and free variables free $(\varphi) \in 2^{\mathrm{Ag} \cup \mathrm{Vr}}$ of

$a$ GRADEDSL formula $\varphi$ is inductively defined as follows:

- $\operatorname{free}(p) \triangleq \emptyset$, where $p \in \operatorname{AP}$;

- $\operatorname{free}(\neg \varphi) \triangleq \operatorname{free}(\varphi)$;

- $\operatorname{free}\left(\varphi_{1} \vee \varphi_{2}\right) \triangleq \operatorname{free}\left(\varphi_{1}\right) \cup \operatorname{free}\left(\varphi_{2}\right)$;

- $\operatorname{free}(X \varphi) \triangleq \operatorname{Ag} \cup \operatorname{free}(\varphi)$;

- $\operatorname{free}\left(\varphi_{1} \cup \varphi_{2}\right) \triangleq \operatorname{Ag} \cup \operatorname{free}\left(\varphi_{1}\right) \cup$ free $\left(\varphi_{2}\right)$;

- free $\left(\left\langle\left\langle x_{1}, \ldots, x_{n}\right\rangle\right\rangle^{\geq g} \varphi\right) \triangleq \operatorname{free}(\varphi) \backslash\left\{x_{1}, \ldots, x_{n}\right\}$;

- $\operatorname{free}((\alpha, x) \varphi) \triangleq \begin{cases}\operatorname{free}(\varphi), & \text { if } \alpha \notin \text { free }(\varphi) ; \\ (\operatorname{free}(\varphi) \backslash\{\alpha\}) \cup\{x\}, & \text { otherwise. }\end{cases}$

A formula $\varphi$ without free agents (resp., variables), i.e., with free $(\varphi) \cap \operatorname{Ag}=\emptyset$ (resp., free $(\varphi) \cap \mathrm{Vr}=\emptyset$ ), is called agent-closed (resp., variable-closed). If $\varphi$ is both agent- and variable-closed, it is called a sentence. 
Roughly, the quantifier rank of $\varphi$ is the maximum, over all paths in the parse-tree of $\varphi$, of the number of strategy quantifiers that appear on the path, e.g., $\left\langle\left\langle x_{1}, \ldots, x_{n}\right\rangle\right\rangle^{\geq g}\left(\alpha_{1}, x_{1}\right) \ldots\left(\alpha_{n}, x_{n}\right) \bigwedge_{i=1}^{n}\left(\langle\langle\mathrm{y}\rangle\rangle\left(\alpha_{i}, \mathrm{y}\right) \psi_{i}\right) \rightarrow \psi_{i}$ has quantifier rank 2 if each $\psi_{i}$ is quantifier free.

Definition 2.3. The quantifier $\operatorname{rank} \operatorname{qr}(\varphi) \in \mathbb{N}$ of a GRADEDSL formula $\varphi$ is inductively defined as follows:

- $\operatorname{qr}(p) \triangleq 0$, where $p \in \mathrm{AP}$;

- $\operatorname{qr}(\mathrm{OP} \varphi) \triangleq \operatorname{qr}(\varphi)$, where $\mathrm{OP} \in\{\neg, \mathrm{X}, b\}$;

- $\operatorname{qr}\left(\varphi_{1} \mathrm{OP} \varphi_{2}\right) \triangleq \max \left(\operatorname{qr}\left(\varphi_{1}\right), \operatorname{qr}\left(\varphi_{2}\right)\right)$ where $\mathrm{OP} \in\{\mathrm{V}, \mathrm{U}\}$

- $\operatorname{qr}\left(\left\langle\left\langle x_{1}, \ldots, x_{n}\right\rangle\right\rangle^{\geq g} \varphi\right) \triangleq \operatorname{qr}(\varphi)+1$.

Roughly, a quantifier-block of $\varphi$ is a maximally-consecutive sequence of quantifiers in $\varphi$ of the same type (i.e., either all existential, or all universal). The quantifier-block rank of a formula is like the quantifier rank except that a quantifier block of $j$ quantifiers contributes 1 instead of $j$ to the count. The formal definition follows:

Definition 2.4. The quantifier-block rank $q b r(\varphi) \in \mathbb{N}$ of a GradedSL formula $\varphi$ that uses the shorthand for universal strategy quantifiers is inductively defined as follows:

- $\operatorname{qbr}(p) \triangleq 0$, where $p \in \mathrm{AP}$;

- $\operatorname{qbr}(\mathrm{OP} \varphi) \triangleq \operatorname{qbr}(\varphi)$, where $\mathrm{OP} \in\{\neg, \mathrm{X}, b\}$;

- $\operatorname{qbr}\left(\varphi_{1} \mathrm{OP} \varphi_{2}\right) \triangleq \max \left(\mathrm{qbr}\left(\varphi_{1}\right), \mathrm{qbr}\left(\varphi_{2}\right)\right)$ where $\mathrm{OP} \in\{\vee, \mathrm{U}\}$;

- $\mathrm{qbr}\left(\left\langle\left\langle x_{1}, \ldots, x_{n}\right\rangle\right\rangle^{\geq g} \varphi\right) \triangleq \operatorname{qbr}(\varphi)$ if $\varphi$ begins with an existential strategy quantifier, and $\mathrm{qbr}(\varphi)+1$ otherwise.

- $\mathrm{qbr}\left(\left[\left[x_{1}, \ldots, x_{n}\right]^{<g} \varphi\right) \triangleq \mathrm{qbr}(\varphi)\right.$ if $\varphi$ begins with a universal strategy quantifier, and $\mathrm{qbr}(\varphi)+1$ otherwise.

Note that we treat $\neg\left\langle\left\langle x_{1}, \ldots, x_{n}\right\rangle\right\rangle^{\geq g} \neg \varphi$ differently to $\left[\left[x_{1}, \ldots, x_{n}\right]^{<g} \varphi\right.$. Thus, one should choose the existential and universal quantifiers judiciously in order to obtain a low quantifier-block rank. 


\subsection{Models}

Sentences of GRADEDSL are interpreted over arenas 3 , just as for ATL and SL [5, 4].

Definition 2.5. An arena over fixed sets of atomic proposition $\mathrm{AP}$ and agents $\mathrm{Ag}$ is a tuple $\mathcal{A} \triangleq\left\langle\mathrm{Ac}, \mathrm{St}, s_{I}\right.$, ap, tr $\rangle$, where:

- Ac is a finite set of actions;

- St is a finite set of states;

- $s_{I} \in \mathrm{St}$ is the initial state;

- ap : St $\rightarrow 2^{\mathrm{AP}}$ is the labeling function mapping each state to the set of atomic propositions true in that state;

- Let $\mathrm{Dc} \triangleq \mathrm{Ag} \rightarrow \mathrm{Ac}$ be the set of decisions, i.e., functions describing the choice of an action by every agent. Then, $\mathrm{tr}: \mathrm{Dc} \rightarrow(\mathrm{St} \rightarrow \mathrm{St})$ is a transition function mapping every decision $\delta \in \mathrm{Dc}$ to a function $\operatorname{tr}(\delta): \mathrm{St} \rightarrow \mathrm{St}$.

We will usually take the set $\mathrm{Ag}$ of agents to be $\left\{\alpha_{1}, \ldots, \alpha_{n}\right\}$. A path (from $s$ ) is a finite or infinite non-empty sequence of states $s_{1} s_{2} \ldots$ such that $s=s_{1}$ and for every $i$ there exists a decision $\delta$ with $\operatorname{tr}(\delta)\left(s_{i}\right)=s_{i+1}$. Given a path $\pi=s_{1} s_{2} \ldots$, with $\lambda(\pi)$ we denote the label of $\pi$ as a sequence of sets of atomic propositions $\pi_{1}, \pi_{2}, \ldots$ where $\operatorname{ap}\left(s_{1}\right)=\pi_{1}, \operatorname{ap}\left(s_{2}\right)=\pi_{2}$, and so on. The set of paths starting with $s$ is denoted $\operatorname{Pth}(s)$. The set of finite paths from $s$, called the histories (from $s$ ), is denoted $\operatorname{Hst}(s)$. A strategy (from $s$ ) is a function $\sigma \in \operatorname{Str}(s) \triangleq \operatorname{Hst}(s) \rightarrow$ Ac that prescribes which action has to be performed given a history. We write Pth, Hst, Str for the set of all paths, histories, and strategies (no matter where they start). We use the standard notion of equality between strategies [35, i.e., $\sigma_{1}=\sigma_{2}$ iff for all $\rho \in$ Hst, $\sigma_{1}(\rho)=\sigma_{2}(\rho)$. This extends to equality between two $n$-tuples of strategies in the natural way, i.e., coordinatewise. There is a subtlety in this definition, i.e., two strategies are different if they differ on some history $\rho$, even if that history is not reachable using either of the strategies.

\subsection{Semantics}

As for SL, the interpretation of a GRADEDSL formula requires a valuation of its free placeholders.

\footnotetext{
${ }^{3}$ This is sometimes called a Concurrent Game Structure.
} 
Definition 2.6. An assignment (from $s$ ) is a function $\chi \in \operatorname{Asg}(s) \triangleq(\operatorname{Vr} \cup \mathrm{Ag}) \rightarrow$ $\operatorname{Str}(s)$ mapping variables and agents to strategies.

We denote by $\chi[e \mapsto \sigma]$, with $e \in \operatorname{Vr} \cup \operatorname{Ag}$ and $\sigma \in \operatorname{Str}(s)$, the assignment that differs from $\chi$ only in the fact that $e$ maps to $\sigma$. Extend this definition to tuples: for $\bar{e}=\left(e_{1}, \ldots, e_{n}\right)$ with $e_{i} \neq e_{j}$ for $i \neq j$, define $\chi[\bar{e} \mapsto \bar{\sigma}]$ to be the assignment that differs from $\chi$ only in the fact that $e_{i}$ maps to $\sigma_{i}$ (for each $i$ ).

Since an assignment ensures that all free variables are associated with strategies, it induces a play.

Definition 2.7. Let $\chi \in \operatorname{Asg}(s)$ be an assignment. $B y(\chi, s)$-play we denote the path $\pi \in \operatorname{Pth}(s)$ such that, for all $i \in \mathbb{N}$, it holds that $\pi_{i+1}=\operatorname{tr}(\mathrm{dc})\left(\pi_{i}\right)$, where $\operatorname{dc}(\alpha) \triangleq \chi(\alpha)\left(\pi_{\leq i}\right)$, for $\alpha \in \mathrm{Ag}$. The function play : Asg $\times \mathrm{St} \rightarrow \mathrm{Pth}$, with $\operatorname{dom}($ play $) \triangleq\{(\chi, s): \chi \in \operatorname{Asg}(s)\}$, maps $(\chi, s)$ to the $(\chi, s)$-play $\operatorname{play}(\chi, s)$ $\in \operatorname{Pth}(s)$.

The notation $\pi_{\leq i}$ (resp. $\pi_{<i}$ ) denotes the prefix of the sequence $\pi$ of length $i$ (resp. $i-1$ ). Similarly, the notation $\pi_{i}$ denotes the $i$ th symbol of $\pi$. Thus, play $(\chi, s)_{i}$ is the $i$ th state on the play determined by $\chi$ from $s$.

The following definition of $\chi_{i}$ says how to interpret an assignment $\chi$ starting from a point $i$ along the play, i.e., for each placeholder $e$, take the action the strategy $\chi(e)$ would do if it were given the prefix of the play up to $i$ followed by the current history.

Definition 2.8. For $\chi \in \operatorname{Asg}(s)$ and $i \in \mathbb{N}$, writing $\rho \triangleq \operatorname{play}(\chi, s)_{\leq i}$ (the prefix of the play up to $i$ ) and $t \triangleq \operatorname{play}(\chi, s)_{i}$ (the last state of $\rho$ ) define $\chi_{i} \in \operatorname{Asg}(t)$ to be the assignment from $t$ that maps $e \in \mathrm{Vr} \cup \mathrm{Ag}$ to the strategy that maps $h \in \operatorname{Hst}(t)$ to the action $\chi(e)\left(\rho_{<i} \cdot h\right)$.

The semantics of GradedSL mimics the one for SL as given in [4. Given an arena $\mathcal{A}$, for all states $s \in \mathrm{St}$ and assignments $\chi \in \operatorname{Asg}(s)$, we now define the relation $\mathcal{A}, \chi, s \models \varphi$, read $\varphi$ holds at $s$ in $\mathcal{A}$ under $\chi$.

Definition 2.9. Fix an arena $\mathcal{A}$. For all states $s \in$ St and assignments $\chi \in$ $\operatorname{Asg}(s)$, the relation $\mathcal{A}, \chi, s \models \varphi$ is defined inductively on the structure of $\varphi$ :

- $\mathcal{A}, \chi, s=p$ iff $p \in \operatorname{ap}(s)$;

- $\mathcal{A}, \chi, s \mid=\neg \varphi$ iff $\mathcal{A}, \chi, s \not \models \varphi ;$

- $\mathcal{A}, \chi, s=\varphi_{1} \vee \varphi_{2}$ iff $\mathcal{A}, \chi, s \models \varphi_{1}$ or $\mathcal{A}, \chi, s=\varphi_{2}$; 
- $\mathcal{A}, \chi, s=\mathrm{X} \varphi$ iff $\mathcal{A}, \chi_{1}, \operatorname{play}(\chi, s)_{1} \models \varphi$;

- $\mathcal{A}, \chi, s \models \varphi_{1} \cup \varphi_{2}$ iff there is $i \in \mathbb{N}$ such that $\mathcal{A}, \chi_{i}$, $\operatorname{play}(\chi, s)_{i} \models \varphi_{2}$ and, for all $j \in \mathbb{N}$ with $j<i$, it holds that $\mathcal{A}, \chi_{j}$, play $(\chi, s)_{j}=\varphi_{1}$;

- $\mathcal{A}, \chi, s \models(\alpha, x) \varphi$ iff $\mathcal{A}, \chi[\alpha \mapsto \chi(x)], s \models \varphi$;

- $\mathcal{A}, \chi, s \models\left\langle\left\langle x_{1}, \ldots, x_{n}\right\rangle\right\rangle^{\geq g} \varphi$ iff there exist $g$ many $n$-tuples of strategies $\overline{\sigma_{i}}$ $(0 \leq i<g)$ such that:

$-\overline{\sigma_{i}} \neq \overline{\sigma_{j}}$ for $i \neq j$;

$-\mathcal{A}, \chi\left[\bar{x} \mapsto \overline{\sigma_{i}}\right], s \mid=\varphi$ for $0 \leq i<g$ and $\bar{x}=\left(x_{1}, \ldots, x_{n}\right)$.

Intuitively, $\left\langle\left\langle x_{1}, \ldots, x_{n}\right\rangle\right\rangle^{\geq g} \varphi$ expresses that the number of distinct tuples of strategies that satisfy $\varphi$ is at least $g$.

As usual, if $\chi$ and $\chi^{\prime}$ agree on $\operatorname{free}(\varphi)$, then $\mathcal{A}, \chi, s=\varphi$ if and only if $\mathcal{A}, \chi^{\prime}, s=\varphi$, i.e., the truth of $\varphi$ does not depend on the values the assignment takes on placeholders that are not free. Thus, for a sentence $\varphi$, we write $\mathcal{A}=\varphi$ to mean that $\mathcal{A}, \chi, s_{I} \models \varphi$ for some (equivalently, for all) assignments $\chi$, and where $s_{I}$ is the initial state of $\mathcal{A}$.

\subsection{Fragments of GRADEDSL}

In this section we introduce various syntactic fragments of GRADEDSL. Obviously SL can be considered a fragment of GRADEDSL: note that the GradedSL quantifier $\left\langle\left\langle x_{1}, \ldots, x_{n}\right\rangle\right\rangle^{\geq g}$ in case $g=1$ and $n=1$ has the same semantics as the SL quantifier $\left\langle\left\langle x_{1}\right\rangle\right\rangle$. The next result shows that GRADEDSL is strictly more expressive than SL, i.e., there is a GrADEDSL sentence whose models are not the set of models of any SL sentence.

Theorem 2.1. GRADEDSL is strictly more expressive than SL.

Proof. Fix $\mathrm{Ag}=\{\alpha\}, \mathrm{AP}=\{p\}, \mathrm{St}=\{s\}, s_{I}=s, \operatorname{ap}(s)=\{p\}$. Define $\mathcal{A}=\left\langle\mathrm{Ac}_{A}\right.$, St, $s_{I}$, ap, $\left.\operatorname{tr}_{A}\right\rangle$, where $\operatorname{Ac}_{A}=\{0\}$, and $\operatorname{tr}_{A}(\delta)(s)=s$ for every decision $\delta$; and $\mathcal{B}=\left\langle\mathrm{Ac}_{B}, \mathrm{St}, s_{I}\right.$, ap, $\left.\operatorname{tr}_{B}\right\rangle$, where $\mathrm{Ac}_{B}=\{0,1\}$, and $\operatorname{tr}_{B}(\delta)(s)=s$ for every decision $\delta$. Thus, each arena consists of a single state with self-loops, the difference being that in $\mathcal{B}$ there are two actions while in $\mathcal{A}$ there is only a single action.

Consider the GradedSL formula $\neg\langle\langle x\rangle\rangle^{\geq 2}$ true. Note that $\mathcal{A}=\neg\langle\langle x\rangle\rangle^{\geq 2}$ true (since there is only a single strategy in $\mathcal{A}$ ), while $\mathcal{B} \not \forall \neg\langle\langle x\rangle\rangle^{\geq 2}$ true (since there are at least two, and in fact $2^{\aleph_{0}}$ many, strategies in $\mathcal{B}$ ).

Let $\chi_{A}$ be the unique assignment in $\mathcal{A}$, i.e., that maps $\alpha$ and every variable in $\mathrm{Vr}$ to the strategy $\sigma$ defined by $\sigma(h)=0$ for all histories $h$. We claim that, for every SL formula $\varphi$, if $\mathcal{A}, \chi_{A}, s=\varphi$ then, for all assignments $\chi$ we have that 
$\mathcal{B}, \chi, s \models \varphi$. Thus, in particular, no SL sentence can distinguish between $\mathcal{A}$ and $\mathcal{B}$, and the theorem follows.

One can easily prove the claim by induction on the structure of an SL formula. Alternatively, one may note that $\mathcal{A}$ and $\mathcal{B}$ are locally-isomorphic, and thus agree on all SL formulas (see [36, Section 3] for the definition and properties of "local-isomorphism") $4^{4}$

Recall that SL has a few natural syntactic fragments, the most powerful of which is $\mathrm{SL}[\mathrm{NG}]$ (here "NG" stands for Nested-Goal). Recall that in $\mathrm{SL}[\mathrm{NG}]$, we require that bindings and quantifications appear in exhaustive blocks. I.e., whenever there is a quantification over a variable in a formula $\psi$ it is part of a consecutive sequence of quantifiers that covers all of the free variables that appear in $\psi$, and whenever an agent is bound to a strategy then it is part of a consecutive sequence of bindings of all agents to strategies. Also, formulas with free agents are not allowed. We define GradedSL[NG] in a similar way, as follows.

A quantification prefix over a set $\mathrm{V} \subseteq \mathrm{Vr}$ of variables is a sequence $\wp$ from the set

$\left\{\left\langle\left\langle x_{1}, \ldots, x_{n}\right\rangle\right\rangle^{\geq g}, \llbracket\left[x_{1}, \ldots, x_{n}\right]^{<g}: n \in \mathbb{N}, x_{1}, \ldots, x_{n} \in \mathrm{V} \wedge g \in \mathbb{N} \cup\left\{\aleph_{0}, \aleph_{1}, 2^{\aleph_{0}}\right\}\right\}^{*}$

such that each $x \in \mathrm{V}$ occurs exactly once in $\wp$. A binding prefix is a sequence $b \in\{(\alpha, x): \alpha \in \mathrm{Ag} \wedge x \in \mathrm{Vr}\}^{*}$ such that each $\alpha \in \mathrm{Ag}$ occurs exactly once in $b$. We denote the set of binding prefixes by Bn, and the set of quantification prefixes over $\mathrm{V}$ by $\mathrm{Qn}(\mathrm{V})$.

Definition 2.10. GRadedSL[NG] formulas are built inductively using the following grammar, with $p \in \mathrm{AP}, \wp \in \mathrm{Qn}(\mathrm{V})(\mathrm{V} \subseteq \mathrm{Vr})$, and $b \in \mathrm{Bn}$ :

$$
\varphi::=p|\neg \varphi| \varphi \vee \varphi|\mathrm{X} \varphi| \varphi \mathrm{U} \varphi|\wp \varphi| b \varphi
$$

where in the rule $\wp \varphi$ we require that $\varphi$ is agent-closed and $\wp \in \operatorname{Qn}($ free $(\varphi))$. In this case we call $\wp \varphi$ a principal formula.

Formulas of GRADEDSL[NG] can be classified according to their alternation number, i.e., the maximum number of quantifier switches in a quantification prefix. 5 Formally:

Definition 2.11. The alternation number alt $(\varphi)$ of a GRADEDSL[NG] formula $\varphi$ is defined as follows:

\footnotetext{
${ }^{4}$ We thank an anonymous reviewer for pointing this out.

${ }^{5}$ In [4. the alternation number is described for all formulas of SL, but only used for the Nested-Goal fragment. Thus, here, we only define it for GradedSL[NG].
} 
- $\operatorname{alt}(p) \triangleq 0$, where $p \in \mathrm{AP}$;

- $\operatorname{alt}(\mathrm{OP} \varphi) \triangleq \operatorname{alt}(\varphi)$, where $\mathrm{OP} \in\{\neg, \mathrm{X}, b\}$;

- $\operatorname{alt}\left(\varphi_{1} \mathrm{OP} \varphi_{2}\right) \triangleq \max \left(\operatorname{alt}\left(\varphi_{1}\right), \operatorname{alt}\left(\varphi_{2}\right)\right)$ where $\mathrm{OP} \in\{\vee, \mathrm{U}\}$;

- $\operatorname{alt}(\wp \varphi) \triangleq \max (\operatorname{alt}(\varphi), \operatorname{alt}(\wp))$ where $\wp=\wp_{1} \ldots \wp|\wp|-1$ is a quantification prefix and $\operatorname{alt}(\wp) \triangleq \sum_{i=1}^{|\wp|-1} \operatorname{switch}\left(\wp_{i}, \wp_{i+1}\right)$, where $\operatorname{switch}\left(Q, Q^{\prime}\right)=0$ if $Q$ and $Q^{\prime}$ are either both universal or both existential quantifiers, and 1 otherwise 6 .

Another important fragment of SL is $\mathrm{SL}[1 \mathrm{G}]$ (here " $1 \mathrm{G}$ " stands for OneGoal). Intuitively, $\mathrm{SL}[1 \mathrm{G}]$ is the fragment of GRADEDSL[NG] in which quantification is immediately followed by binding. The importance of this fragment stems from the fact that it strictly includes $\mathrm{ATL}^{\star}$ while maintaining the same complexity for both the model checking and the satisfiability problems, i.e. 2ExpTime-complete [32, 4. However, it is commonly believed that Nash Equilibrium cannot be expressed in this fragment. Similarly, we give the following definition of GRADEDSL[1G]:

Definition 2.12. GRAdedSL[1G] formulas are built inductively using the following grammar, with $p \in \mathrm{AP}, \wp \in \mathrm{Qn}(\mathrm{V})(\mathrm{V} \subseteq \mathrm{Vr})$, and $b \in \mathrm{Bn}$ :

$$
\varphi::=p|\neg \varphi| \varphi \vee \varphi|\mathrm{X} \varphi| \varphi \mathrm{U} \varphi \mid \wp b \varphi
$$

where $\wp$ is a quantification prefix over free $(b \varphi)$.

Finally, an important fragment (in which one can express uniqueness of strategy profiles) is when all grades are in $\mathbb{N}$.

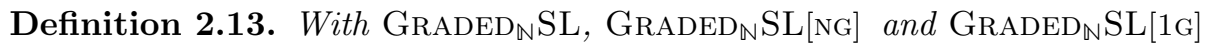
we refer for the fragments in which all grades are from the set $\mathbb{N}$.

\section{Model-checking GRADEDSL}

In this section we study the model-checking problem for GRADEDSL and show that it is decidable with a time-complexity that is non-elementary (i.e., not bounded by any fixed tower of exponentials). However, it is elementary if the number of blocks of quantifiers is fixed.

\footnotetext{
${ }^{6}$ Observe that formulas of the form $\wp \varphi$ have no free variables and thus one cannot form formulas of the form $\wp^{\prime} \wp \varphi$.
} 
Definition 3.1. The model-checking problem for GRADEDSL (respectively, GRADEDSL[NG]) is the following decision problem: given a formula $\varphi$ from GradedSL (respectively, GradedSL[NG]) over some finite sets of atoms AP, agents $\mathrm{Ag}$, and variables $\mathrm{Vr}$, and given an arena $\mathcal{A}$ over the sets $\mathrm{AP}$ and $\mathrm{Ag}$, decide whether $\mathcal{A}=\varphi$.

When measuring computational complexity, the grades in formulas are written in unary.

For the algorithmic procedures, we follow an automata-theoretic approach [37, reducing the decision problem for the logic to the emptiness problem of an automaton. The procedure we propose here extends that used for SL in 44. The only case that is different is the new graded quantifier over tuples of strategies, i.e., we show how to convert a GRADEDSL formula $\varphi$ into an automaton that accepts exactly the (tree encodings) of the assignments that satisfy $\varphi$.

Tree Automata. A $\Sigma$-labeled $\Upsilon$-tree $\mathrm{T}$ is a pair $\langle T, V\rangle$ where $T \subseteq \Upsilon^{+}$is prefix-closed (i.e., if $t \in T$ and $s \in \Upsilon^{+}$is a prefix of $t$ then also $s \in T$ ), and $V: T \rightarrow \Sigma$ is a labeling function. Note that every word $w \in \Upsilon^{+} \cup \Upsilon^{\omega}$ with the property that every prefix of $w$ is in $T$, can be thought of as a path in T. Infinite paths are called branches. Nondeterministic tree automata (NTA) are a generalization to infinite trees of the classical automata on words 38 . Alternating tree automata (ATA) are a further generalization of nondeterministic tree automata 39. Intuitively, on visiting a node of the input tree, while an NTA sends exactly one copy of itself to each of the successors of the node, an ATA can send several copies to the same successor. We use the parity acceptance condition 37 .

For a set $X$, let $\mathrm{B}^{+}(X)$ be the set of positive Boolean formulas over $X$, including the constants true and false. A set $Y \subseteq X$ satisfies a formula $\theta \in$ $\mathrm{B}^{+}(X)$, written $Y \models \theta$, if assigning true to elements in $Y$ and false to elements in $X \backslash Y$ makes $\theta$ true.

Definition 3.2. An Alternating Parity Tree-Automaton (APт) is a tuple $\mathcal{M} \triangleq$ $\left\langle\Sigma, \Delta, \mathrm{Q}, \delta, q_{\mathrm{o}}, \mathrm{F}\right\rangle$, where

- $\Sigma$ is the input alphabet,

- $\Delta$ is a set of directions,

- $\mathrm{Q}$ is a finite set of states,

- $q_{\mathrm{o}} \in \mathrm{Q}$ is an initial state,

- $\delta: \mathrm{Q} \times \Sigma \rightarrow \mathrm{B}^{+}(\Delta \times \mathrm{Q})$ is an alternating transition function, and 
- $\mathrm{F}$, an acceptance condition, is of the form $\left(\mathrm{F}_{1}, \ldots, \mathrm{F}_{k}\right) \in\left(2^{\mathrm{Q}}\right)^{+}$where $\mathrm{F}_{1} \subseteq \mathrm{F}_{2} \ldots \subseteq \mathrm{F}_{k}=\mathrm{Q}$.

The set $\Delta \times \mathrm{Q}$ is called the set of moves. An NTA is an ATA in which each conjunction in the transition function $\delta$ has exactly one move $(d, q)$ associated with each direction $d$.

An input tree for an ApT is a $\Sigma$-labeled $\Delta$-tree $\mathrm{T}=\langle\mathrm{T}, \mathrm{v}\rangle$. A run of an APT on an input tree $\mathrm{T}=\langle\mathrm{T}, \mathrm{v}\rangle$ is a $(\Delta \times \mathrm{Q})$-tree $\mathrm{R}$ such that, for all nodes $x \in \mathrm{R}$, where $x=\left(d_{1}, q_{1}\right) \ldots\left(d_{n}, q_{n}\right)$ (for some $n \in \mathbb{N}$ ), it holds that (i) $y \triangleq$ $\left(d_{1}, \ldots, d_{n}\right) \in \mathrm{T}$ and (ii) there is a set of moves $\mathrm{S} \subseteq \Delta \times \mathrm{Q}$ with $\mathrm{S} \models \delta\left(q_{n}, \mathrm{v}(y)\right)$ such that $x \cdot(d, q) \in \mathrm{R}$ for all $(d, q) \in \mathrm{S}$.

The acceptance condition allows us to say when a run is successful. Let $\mathrm{R}$ be a run of an Apt $\mathcal{M}$ on an input tree T and $u \in(\Delta \times \mathrm{Q})^{\omega}$ one of its branches. Let $\inf (u) \subseteq \mathrm{Q}$ denote the set of states that occur in infinitely many moves of $u$. Say that $u$ satisfies the parity acceptance condition $\mathrm{F}=\left(\mathrm{F}_{1}, \ldots, \mathrm{F}_{k}\right)$ if the least index $i \in[1, k]$ for which $\inf (u) \cap \mathrm{F}_{i} \neq \emptyset$ is even. A run is successful if all its branches satisfy the parity acceptance condition F. An APT accepts an input tree $\mathrm{T}$ iff there exists a successful run $\mathrm{R}$ of $\mathcal{M}$ on $\mathrm{T}$.

The language $\mathrm{L}(\mathcal{M})$ of the APT $\mathcal{M}$ is the set of trees $\mathrm{T}$ accepted by $\mathcal{M}$. Two automata are equivalent if they have the same language. The emptiness problem for alternating parity tree-automata is to decide, given $\mathcal{M}$, whether $\mathrm{L}(\mathcal{M})=\emptyset$. The universality problem is to decide whether $\mathcal{M}$ accepts all trees.

\subsection{From Logic to Automata}

We reduce the model-checking problem of GRADEDSL to the emptiness problem for alternating parity tree automata 4. The main step is to translate every GradedSL formula $\varphi$ (i.e., $\varphi$ may have free placeholders), arena $\mathcal{A}$, and state $s$, into an APT that accepts a tree if and only if the tree encodes an assignment $\chi$ such that $\mathcal{A}, \chi, s=\varphi$.

We first describe the encoding, following 4. Informally, the arena $\mathcal{A}$ is encoded by its "tree-unwinding starting from $s$ " whose nodes represent histories, i.e., the St-labeled St-tree $\mathbf{T} \triangleq\langle\operatorname{Hst}(s), \mathbf{u}\rangle$ such that $\mathbf{u}(h)$ is the last symbol of $h$. Then, every strategy $\chi(e)$ with $e \in$ free $(\varphi)$ is encoded as an Ac-labelled tree over the unwinding. The unwinding and these strategies $\chi(e)$ are viewed as a single $(\mathrm{VAL} \times \mathrm{St})$-labeled tree where $\mathrm{VAL} \triangleq \operatorname{free}(\varphi) \rightarrow \mathrm{Ac}$.

Definition 3.3. The encoding of $\chi$ (w.r.t. $\varphi, \mathcal{A}, s)$ is the (VAL $\times \mathrm{St}$ )-labeled St-tree $\mathrm{T} \triangleq\langle\mathrm{T}, \mathrm{u}\rangle$ such that $\mathrm{T}$ is the set of histories $h$ of $\mathcal{A}$ starting with $s$ and $\mathrm{u}(h) \triangleq(\mathrm{f}, q)$ where $q$ is the last symbol in $h$ and $\mathrm{f}:$ free $(\varphi) \rightarrow$ Ac is defined by $\mathrm{f}(e) \triangleq \chi(e)(h)$ for all $e \in$ free $(\varphi)]^{7}$

\footnotetext{
${ }^{7}$ In case free $(\varphi)=\emptyset$, then $\mathrm{f}$ is the (unique) empty function. In this case, the encoding of every $\chi$ may be viewed as the tree-unwinding from $s$.
} 
We now state and prove a lemma that says one can translate every formula in GRADEDSL into an APT. It is proved by induction on the structure of the formula $\varphi$, as in [4]. The idea for handling the new case, i.e., the graded quantifier $\left\langle\left\langle x_{1}, \ldots, x_{n}\right\rangle\right\rangle{ }^{\geq g} \psi$, is to build an APT that is a projection of an APT that itself checks that each of the $g$ tuples of strategies satisfies $\psi$ and that each pair of $g$ tuples is distinct. The case that $g \in \mathbb{N}$ directly builds the required automaton (as is done for SL [4]), while the case that $g \in\left\{\aleph_{0}, \aleph_{1}, 2^{\aleph_{0}}\right\}$ goes through logic. Write MSOL for monadic second-order logic in the signature of trees. We use the following two results that show how to express counting quantifiers in MSOL. The first is due to Rabin 2.9 and is nicely exposed in [38.

Theorem 3.1 (MSOL and Automata). For every MSOL formula $\alpha(Y)$ there exists an APT accepting the set of trees $Y$ such that $\alpha(Y)$ holds. Conversely, for every APT there is an MSOL formula $\alpha(Y)$ that holds on those $Y$ that are accepted by the APT.

The second is due to [40] and is proved using the composition technique.

Theorem 3.2. For every $M S O L$ formula $\alpha(\bar{X}, Y)$ and $\kappa \in\left\{\aleph_{0}, \aleph_{1}, 2^{\aleph_{0}}\right\}$ there exists an MSOL formula $\beta(\bar{X})$ equivalent to "there exist $\kappa$ many trees $Y$ such that $\alpha(\bar{X}, Y)$ ".

Lemma 3.1. For every GRADEDSL formula $\varphi$, arena $\mathcal{A}$, and state $s \in \mathrm{St}$, there exists an APT $\mathcal{M}$ such that for all assignments $\chi$, if $\mathrm{T}$ is the encoding of $\chi$ (w.r.t. $\varphi, \mathcal{A}, s)$, then $\mathcal{A}, \chi, s \mid=\varphi$ iff $\mathrm{T} \in \mathrm{L}(\mathcal{M})$.

Proof. As in [4] we induct on the structure of the formula $\varphi$ to construct the corresponding automaton $\mathcal{M}$. The Boolean operations are easily dealt with using the fact that disjunction corresponds to non-determinism, and negation corresponds to dualising the automaton. The temporal operators are dealt with by following the unique play (determined by the given assignment) and verifying the required subformulas, e.g., for $\mathbf{X} \psi$ the automaton, after taking one step along the play, launches a copy of the automaton for $\psi$. All of these operations incur a linear blowup in the size of the automaton. The only case that differs from SL is the quantification, i.e., we need to handle the case that $\varphi=\left\langle\left\langle x_{1}, \ldots, x_{n}\right\rangle\right\rangle^{\geq g} \psi$. Recall that $\mathcal{G}, \chi, s \models\left\langle\left\langle x_{1}, \ldots, x_{n}\right\rangle\right\rangle^{\geq g} \psi$ iff there exists $g$ many tuples $\overline{\sigma_{i}}$ of strategies such that: $\overline{\sigma_{a}} \neq \overline{\sigma_{b}}$ for $a \neq b$, and $\mathcal{G}, \chi\left[\bar{x} \mapsto \overline{\sigma_{i}}\right], s \models$ $\psi$ for $0 \leq i<g$.

There are two cases.

Case $g \in\left\{\aleph_{0}, \aleph_{1}, 2^{\aleph_{0}}\right\}$. Consider $\left\langle\left\langle x_{1}, \ldots, x_{n}\right\rangle\right\rangle^{\geq g} \psi$. By induction, there is an Apt $\mathcal{D}$ for $\psi$. Apply Theorem 3.1 to translate $\mathcal{D}$ into an MSOL formula $\alpha$, then apply Theorem 3.2 to get an MSOL formula $\beta$ that holds iff "there exist 
$g$ many tuples of trees such that $\alpha$ " (recall that a tuple of trees is coded as a single tree). Finally, apply Theorem 3.1 to convert $\beta$ into the required APT.

Note that the blowup in the translations (MSOL to APT, and closure under "there exists $\kappa$ many trees") is non-elementary.

Case $g \in \mathbb{N}$. Let $\mathcal{M}$ be the APT for $\psi$, given by induction. We show how to build an NPT for $\varphi$ that mimics the definition of $\varphi$ : it will be a projection of an APT, which itself is the intersection of two automata, one checking that each of the $g$ tuples of strategies satisfies $\psi$, and the other checking that each pair of the $g$ tuples of strategies is distinct.

In more detail, introduce a set of fresh variables $X \triangleq\left\{x_{i}^{j}: i \leq n, j \leq g\right\}$, and consider the formulas $\psi^{j}$ (for $j \leq g$ ) formed from $\psi$ by renaming $x_{i}$ (for $i \leq n)$ to $x_{i}^{j}$. Define $\psi^{\prime} \triangleq \wedge_{j \leq g} \psi^{j}$. Note that, by induction, each $\psi^{j}$ has a corresponding APT and thus, there is an APT $\mathcal{B}_{\mathcal{M}, X}$ for $\psi^{\prime}$ (conjunction can be dealt using universal-choice). Note that the input alphabet for $\mathcal{B}_{\mathcal{M}, X}$ is $\left(\right.$ free $\left(\psi^{\prime}\right) \rightarrow$ Ac) $\times$ St and that $X \subseteq$ free $\left(\psi^{\prime}\right)$.

On the other hand, let $\mathcal{C}_{X}$ be an APT with input alphabet $\left(\right.$ free $\left(\psi^{\prime}\right) \rightarrow$ Ac) $\times$ St that accepts a tree $\mathrm{T}=\langle\mathrm{T}, \mathrm{v}\rangle$ if and only if for every $a \neq b \leq g$ there exists $i \leq n$ and $h \in \mathrm{T}$ such that $\mathrm{v}(h)=(f, q)$ (where $q$ is the last symbol of $h$ ) and $f\left(x_{i}^{a}\right) \neq f\left(x_{i}^{b}\right)$. To build $\mathcal{C}_{X}$ simply form the conjunction of automata $\mathcal{C}_{X}^{a, b}$ (for $a \neq b \leq g$ ), each of which is the disjunction of automata $\mathcal{C}_{X}^{a, b, i}$ (for $i \leq n$ ) that checks (by nondeterministically guessing a path) that there exists a history $h$ starting in $s$ such that $f\left(x_{i}^{a}\right) \neq f\left(x_{i}^{b}\right)$ (where the first co-ordinate of $\mathrm{v}(h)$ is $f)$.

Form the Apt $\mathcal{D}_{\mathcal{M}, X}$ for the intersection of $\mathcal{B}_{\mathcal{M}, X}$ and $\mathcal{C}_{X}$ (formed using universal-choice).

Now, using the classic transformation [41, we remove alternation from the Apt $\mathcal{D}_{\mathcal{M}, X}$ to get an equivalent Npt $\mathcal{N}$ (note that this step costs an exponential). Finally, use the fact that NPTs are closed under projection (with no blowup) to get an NPT for the language $\operatorname{proj}_{X}(L(\mathcal{N}))$ of trees that encode assignments $\chi$ satisfying $\varphi$.

For completeness we recall this last step. If $L$ is a language of $\Sigma$-labeled trees with $\Sigma \triangleq \mathrm{A} \rightarrow \mathrm{B}$, and $X \subset \mathrm{A}$, then the X-projection of $L$, written $\operatorname{proj}_{\mathrm{X}}(L)$, is the language of $\Sigma^{\prime}$-labeled trees with $\Sigma^{\prime} \triangleq \mathrm{A} \backslash \mathrm{X} \rightarrow \mathrm{B}$ such that $\mathrm{T} \triangleq\langle\mathrm{T}, \mathrm{v}\rangle \in \operatorname{proj}_{\mathrm{X}}(L)$ if and only if there exists an X-labeled tree $\langle\mathrm{T}, \mathrm{w}\rangle$ such that the language $L$ contains the tree $\langle\mathrm{T}, \mathrm{u}\rangle$ where $\mathrm{u}: \mathrm{T} \rightarrow(\mathrm{A} \rightarrow \mathrm{B})$ maps $t \in \mathrm{T}$ to $\mathrm{v}(t) \cup \mathrm{w}(t)$. Now, if $\mathcal{N}$ is an NPT with input alphabet $\Sigma \triangleq \mathrm{A} \rightarrow \mathrm{B}$, and if $\mathrm{X} \subset \mathrm{A}$, then there is an NPT with input alphabet $\Sigma^{\prime} \triangleq \mathrm{A} \backslash \mathrm{X} \rightarrow \mathrm{B}$ with language $\operatorname{proj}_{\mathrm{X}}(L(\mathcal{N}))$.

The proof that the construction is correct is immediate.

We now analyse the number of states of the constructed Apt. All the cases in the induction incur at most a linear blowup except for the quantification case. For the quantification case, in case $g \in\left\{\aleph_{0}, \aleph_{1}, 2^{\aleph_{0}}\right\}$ the blowup is nonelementary.

In case $g \in \mathbb{N}$ then the translation incurs an exponential blowup. Indeed, 
the number of states of the APT $\mathcal{B}_{\mathcal{M}, X}$ is $g \times n$ times the number of states of the APT for $\psi$, and since $\mathcal{C}_{X}$ consists of the conjunction of $g(g-1)$ automata (one for each pair of tuples), and each such automaton has $O(n)$ many states, the number of states of $\mathcal{C}_{X}$ is $O\left(n g^{2}\right)$. Thus, the number of states of the APT $\mathcal{D}_{\mathcal{M}, X}$ is polynomial in the number of states of the APT for $\psi$. Finally, the translation from an APT to an NPT results in an exponentially larger automaton 37.

In case all grades are from $\mathbb{N}$ and the formulas are written using the universalstrategy quantifier shorthand, we can easily modify the construction to handle quantifier-blocks in one shot as if they were a single quantifier, i.e., with a single exponential blowup. For instance, suppose $\phi=\left\langle\left\langle y_{1}, \ldots, y_{m}\right\rangle\right\rangle^{\geq h}\left\langle\left\langle x_{1}, \ldots, x_{n}\right\rangle\right\rangle{ }^{\geq g} \psi$ (additional quantifiers are treated similarly). As in the proof, let $\mathcal{M}$ be the APT for $\psi$ and take $\mathcal{D}_{\mathcal{M}, X}$. Now, instead of immediately removing alternation and projecting, build $\mathcal{D}_{\mathcal{M}^{\prime}, Y}$ where $\mathcal{M}^{\prime}$ is $\mathcal{D}_{\mathcal{M}, X}$ and $Y \triangleq\left\{y_{i}^{j}: i \leq m, j \leq h\right\}$. Finally, remove alternation from $\mathcal{D}_{\mathcal{M}^{\prime}, Y}$ to get an $\mathrm{NPT}_{\mathcal{N}}^{\prime}$, and then apply the $(X \cup Y)$-projection to the language of $\mathcal{N}^{\prime}$ to get the desired APT for $\phi$. Note that the size of $\mathcal{D}_{\mathcal{M}^{\prime}, Y}$ is exponential in the number of states of $\mathcal{M}$ since the costly step of removing alternation is performed only once. Similarly, to deal with a block of universal strategy quantifiers simply use dualisation. For instance, to deal with $\phi=\left[\left[y_{1}, \ldots, y_{m}\right]^{<h}\left[\left[x_{1}, \ldots, x_{n}\right]^{<g} \psi\right.\right.$ apply the previous procedure to the equivalent formula $\neg\left\langle\left\langle y_{1}, \ldots, y_{m}\right\rangle\right\rangle^{\geq h}\left\langle\left\langle x_{1}, \ldots, x_{n}\right\rangle\right\rangle{ }^{\geq g} \neg \psi$ (recall that negating an APT is done by dualisation, which incurs no blowup).

Theorem 3.3. The model-checking problem for GRADEDSL $\varphi$ is decidable.

Precisely, we have:

1. The complexity is not bounded by any fixed tower of exponentials.

2. The complexity is PTIME-COMPLETE w.r.t. the size of the model.

3. If all grades in $\varphi$ are restricted to be in $\mathbb{N}$, then:

(a) the model-checking problem is in $(k+1)$ ExPTIME if $k \geq 1$ is the quantifier-block rank of $\varphi$.

(b) if $\varphi$ is the form $\wp \psi$, where $\wp$ is a quantifier-block, and $\psi$ is of quantifier-block rank $k-1$, then the model-checking problem is in $k$ ExPTIME.

(c) if $\varphi$ is of the form $\left\langle\left\langle x_{1}, \ldots, x_{n}\right\rangle\right\rangle^{\geq g} \psi$ or $\left[\left[x_{1}, \ldots, x_{n}\right]\right]^{<g} \psi$ then the model-checking problem is in ExPTIME w.r.t. the parameter $g$ (written in unary).

Proof. The lower bounds already hold for SL 4]. For decidability, use Lemma 3.1 to transform the arena and $\varphi$ into an APT and test its emptiness. For the upper bound in item 2, use the fact that the membership problem for 
APT is in PTIME in the number of states. For item 3(a), proceed as follows. The complexity of checking emptiness (resp. universality) of an APT is in ExPTime in the number of states [37. As discussed after Lemma 3.1. for the case that all grades are in $\mathbb{N}$, the number of states of the APT is a tower of exponentials whose height is the quantifier-block rank of $\varphi$. This gives the $(k+1)$ ExPTImE bound. Finally, suppose that $\varphi=\wp \psi$ where $\wp$ consists of, say, $n$ existential quantifiers (resp. universal quantifiers). The quantifier-block rank of $\psi$ is $k-1$. Moreover, the APT $\mathcal{D}_{\psi}$, whose number of states is non-elementary in $k-1$, has the property that it is non-empty (resp. universal) if and only if the arena satisfies $\wp \psi$. Conclude that model checking $\wp \psi$ can be solved in $k$ ExPTIME. For item 3(c), first observe that the size of the APT constructed in Lemma 3.1 grows quadratically in $g$. The statement follows by recalling that the complexity of model-checking formulas of this form is exponential in the number of states of the APT.

Theorem 3.4. The model-checking problem for GRADEDSL[NG] when it is restricted to formulas of maximum alternation number $k$ and grades in $\mathbb{N}$, is $(k+1)$ ExpTime.

Proof. The lower bound already holds for $\mathrm{SL}[\mathrm{NG}]$ [4. For the upper bound, as for $\mathrm{SL}[\mathrm{NG}]$, note that principal formulas are "state formulas", i.e., their truth value only depends on the state in which they are interpreted (this is because they have no free placeholders). Thus, one can replace the general algorithm in Lemma 3.1 with the following marking algorithm. Bottom up, for every principal subformula $\varphi=\wp \psi$ and state $s$ of $\mathcal{A}$, mark $s$ by the truth value of $\mathcal{A}, \chi, s \models \varphi$ (for some, equivalently all, assignments $\chi$ ). Consider these markings as new atomic propositions. Observe that the complexity of marking a state is at most $(k+1)$ ExpTime (by repeatedly applying Theorem 3.3 part 3.). Also, the cost of the whole marking algorithm is the sum of the costs of all the marking rounds, and the number of rounds is at most the size of the formula. Thus the total time is at most $(k+1)$ ExPTime.

We conclude this section with the complexity of the model checking problem for $\operatorname{GRADED}_{\mathbb{N}} \mathrm{SL}[1 \mathrm{G}]$. In this case one can derive the lower bound from the one holding for the corresponding sub-logic in SL (i.e., SL[1G]) and the upper bound by using the same algorithm for $\mathrm{SL}[1 \mathrm{G}]$ but using the (no more complex) construction for the strategic quantifier $\left\langle\left\langle x_{1}, \ldots, x_{n}\right\rangle\right\rangle^{\geq g}$ instead of $\langle\langle x\rangle\rangle$. Indeed the model checking problem for GRADED $\mathrm{N} S[1 \mathrm{G}]$ is 2ExPTIME-COMPLETE. It is worth recalling that $\mathrm{SL}[1 \mathrm{G}]$ strictly subsumes $\mathrm{ATL}^{\star}$ [4]. It is quite immediate to see that this also holds in the graded setting (note that $\mathrm{ATL}^{\star}$ already allows quantifying over tuples of agents' (bound) strategies). As the model checking for $\mathrm{ATL}^{\star}$ is already 2ExPTIME-HARD, we get that also for the graded extension for this logic, which we name GATL ${ }^{\star}$, the model checking problem is 2ExPTIMECOMPLETE. The model checking results for both GATL ${ }^{\star}$ and GRADEDSL[1G] 
are reported in the following theorem.

Theorem 3.5. The model-checking problem for $\mathrm{GATL}^{\star}$ and $\mathrm{GRADED}_{\mathbb{N}} \mathrm{SL}[1 \mathrm{G}]$

is PTIME-COMPLETE w.r.t. the size of the model and 2EXPTIME-COMPLETE w.r.t. the size of the formula.

\section{Analysing Games using GradedSL}

In this section we describe how to use the models and formulas of GRADEDSL to reason about solution concepts from game theory. In particular, we show how to use arenas to model games of finite or infinite duration, and GRADEDSL to express the uniqueness of winning strategies, Nash equilibria, subgame-perfect equilibria, and Pareto-efficient profiles. In all cases this is not more expensive than merely deciding the existence of winning strategies with LTL-goals, i.e., 2EXPTIME.

\subsection{Strategic Form and Infinitely Repeated Games}

The Strategic Form is the most familiar representation of strategic interactions in Game Theory. A game written in this way amounts to a representation of every player's preference for every state of the world, in the special case where states of the world depend only on the players' combined actions.

Definition 4.1. A strategic form game is a tuple $\left(N, A,\left(\preceq_{i}\right)_{i \in N}\right)$, where:

- $N$ is a finite set of $n$ players, indexed by $i$;

- $A=A_{1} \times \ldots \times A_{n}$, where $A_{i}$ is a finite set of actions available to player i. Each vector $a=\left(a_{1}, \ldots, a_{n}\right) \in A$ is called an action profile;

- each $\preceq_{i}$ is a total pre-order (i.e., reflexive and transitive) on $A$.

Note that a common way to give the preference relation is by using a payoff function pay $: A \rightarrow \mathbb{R}$, which assigns a real number to every element in $A$. In this case, the preference relation $\preceq_{i}$ is defined by having $a \preceq_{i} a^{\prime}$ iff $\operatorname{pay}(a) \leq \operatorname{pay}\left(a^{\prime}\right)$.

A classic way to model players that repeatedly interact with each other in a game $\left(N, A,\left(\preceq_{i}\right)_{i \in N}\right)$ is by infinitely repeated games, see e.g., [42. We will illustrate by formalising an iterated prisoner's dilemma (Section 4.3). 


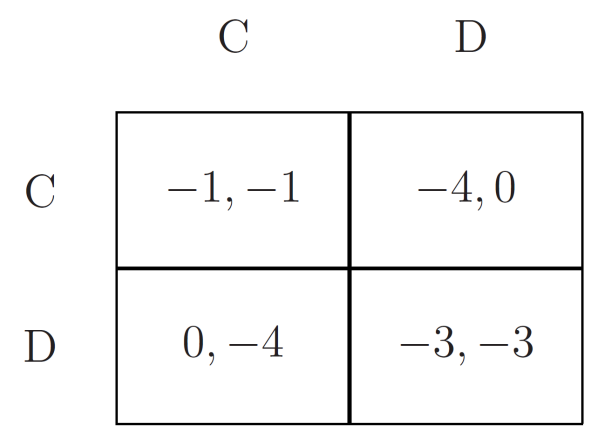

Figure 1: Prisoner's Dilemma in Strategic Form. Each row corresponds to a possible action for player 1, each column corresponds to a possible action for player 2, and each cell corresponds to one possible outcome. Payoffs of the players for an outcome are written in the corresponding cell, with the payoff of player 1 listed first.

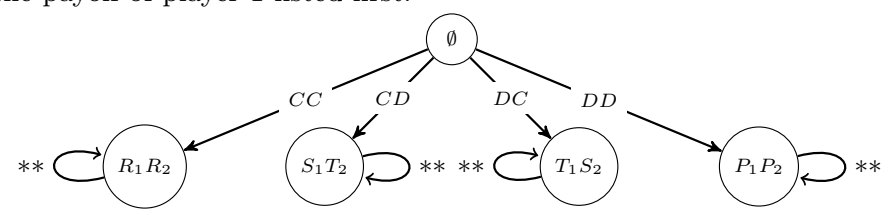

Figure 2: Arena of the Prisoner's dilemma.

\subsection{Quasi-Quantitative Games and Objective-LTL Games}

As expected, we can also specify games by arenas and a payoff function on plays. In this section we define quasi-quantitative games, a generalisation of objective-LTL games $[8$.

Let $\mathcal{A}$ be an arena with $n$ agents. Let $m \in \mathbb{N}$, for each agent $\alpha_{i} \in \mathrm{Ag}$, a quasiquantitative objective is a tuple $S_{i} \triangleq\left\langle\mathrm{f}_{i}, L_{i}^{1}, \ldots, L_{i}^{m}\right\rangle$, where $\mathrm{f}_{i}:\{0,1\}^{m} \rightarrow \mathbb{Z}$, and each $L_{i}^{j}$ is a set of sequences of sets of atomic propositions. If $\pi$ is an infinite path, then agent $\alpha_{i}$ receives payoff $\mathrm{f}_{i}\left(b_{i}^{\pi}\right) \in \mathbb{N}$ where the $j^{\prime}$ th bit of $b_{i}^{\pi}$ is 1 if and only if $\operatorname{ap}(\pi) \in L_{i}^{j}$. We assume agents are trying to maximise their payoffs. The tuple $\mathcal{G}=\left\langle\mathcal{A}, S_{1}, \ldots, S_{n}\right\rangle$ is called a quasi-quantitative game. In case each $f_{i}:\{0,1\}^{m} \rightarrow\{-1,1\}$ we say that the game is win/lose. If $\sum_{1 \leq i \leq n} f_{i}\left(b_{i}^{\pi}\right)=0$ for all $\pi$, then $\mathcal{G}$ is zero-sum, otherwise it is a non zero-sum.

In case each $L_{i}^{j}$ is the set of models of an LTL formula $\varphi_{i}^{j}$ over AP, we call $\mathcal{G}$ an objective-LTL game. We introduce the following useful shorthand. For $\alpha_{i} \in \mathrm{Ag}$ and $a \in\{0,1\}^{m}$, define $\eta_{i}^{a}$ for the LTL formula $\bigwedge_{j \leq m} \psi_{a, j}$ where $\psi_{a, j}=\varphi_{i}^{j}$ if $a_{j}=1$ and $\psi_{a, j}=\neg \varphi_{i}^{j}$ if $a_{j}=0$.

\subsection{Example: The Prisoner's Dilemma (PD)}

A natural way to draw strategic form games is via an n-dimensional matrix. Figure 1 contains the matrix of the classic Prisoner's Dilemma.

The two actions are $C$ (co-operate) and $D$ (defect). The payoffs are the prison sentences (in years) that the prisoners get for each pair of actions that 
they choose 8

The deal is that if both confess then they each get a reduced sentence. If both is offered the choice to confess or remain silent. captured thieves are suspected of Observe that the actual numbers are not important, and that the important thing is the induced preference relation $\mathbf{T}_{i}>\mathbf{R}_{i}>\mathbf{P}_{i}>\mathbf{S}_{i}$, where $\mathbf{R}_{i}$ represents the reward that $\alpha_{i}$ receives if both cooperate; $\mathbf{P}_{i}$ is the punishment that $\alpha_{i}$ receives if both defect; $\mathbf{T}_{i}$ is the temptation that $\alpha_{i}$ receives as a sole defector, and $\mathbf{S}_{i}$ is the sucker payoff that $\alpha_{i}$ receives as a sole cooperator.

We can describe the Prisoner's Dilemma with the arena in Figure 2 and, for agent $\alpha_{i}$, the objective $S_{i} \triangleq\left\langle\mathrm{f}_{i}, \varphi_{i}^{1}, \varphi_{i}^{2}, \varphi_{i}^{3}, \varphi_{i}^{4}\right\rangle$ where $\varphi_{i}^{1} \triangleq \mathbf{X} \mathbf{S}_{i}, \varphi_{i}^{2} \triangleq \mathbf{X} \mathbf{P}_{i}$, $\varphi_{i}^{3} \triangleq \mathbf{X} \mathbf{R}_{i}$, and $\varphi_{i}^{4} \triangleq \mathbf{X} \mathbf{T}_{i}$ and $\mathbf{f}_{i}$ returns the value of its input vector interpreted as a binary number, e.g., $\mathrm{f}_{i}(0100)=4$ that represents the payoff in which $\varphi_{i}^{3}$ is true. In words, we have two agents $\alpha_{1}$ and $\alpha_{2}$. Each agent has two actions, $C$ and $D$. For each possible pair of moves, the game goes in a state whose atomic propositions represent the preferences.

It is well known that in the Prisoner's Dilemma the only Nash equilibrium is for both players to defect. The reason is that each prisoner must hedge against the possibility of the other one defecting. However, it is clear that if they would have both cooperated, they would be better off. If there was a way for one prisoner to later punish a defection of the other prisoner, it may not have to hedge, and would be able to cooperate instead. Such behaviours emerge as a rational choice, for example, when one considers the infinitely repeated prisoner's dilemma, in which the prisoners repeat the basic strategic form game infinitely often $\sqrt{9}$ Indeed, it is well known that for this iterated game (for example, with a payoff that is the mean-payoff of the prison sentences [43]), a new Nash equilibrium emerges, in which both players use the so called Grim strategy, in which a prisoner cooperates as long as the other prisoner cooperates, but switches to always defect the first time the other prisoner defects. Observe that the resulting infinite play of this Nash equilibrium has both players cooperating all the time. The core reason that the pair of grim strategies is a Nash equilibrium for the mean-payoff version of the iterated prisoner's dilemma is that this payoff ignores the price of being a 'sucker' on any finite prefix of the play, i.e., that the mean-payoff of a play is independent of any finite prefix of that play - other properties of the mean are not needed, and constitute unimportant noise. In-

\footnotetext{
${ }^{8}$ The story behind the dilemma is this: Two people have been arrested for robbing a bank and placed in separate isolation cells. Each has two possible choices, remaining silent (action $C$ ) or confessing (action $D$ ). If a robber confesses and the other remains silent, the former is released and the latter stays in prison for a long time. If both confess they are both convicted, but will get early parole. If both remain silent, they get a lighter sentence (e.g., on firearms possession charges). The dilemma faced by the prisoners is that, whatever the choice of the other prisoner, each is better off confessing than remaining silent. But the result obtained when both confess is worse than if they both remain silent.

${ }^{9}$ Alternatively, one can introduce the threat of a punishment for defecting by considering a probabilistic version in which it is unclear to the prisoners how many repetitions will be used. Note, however, that a fixed number of repetitions turns out to be essentially the same as playing only once 43 .
} 


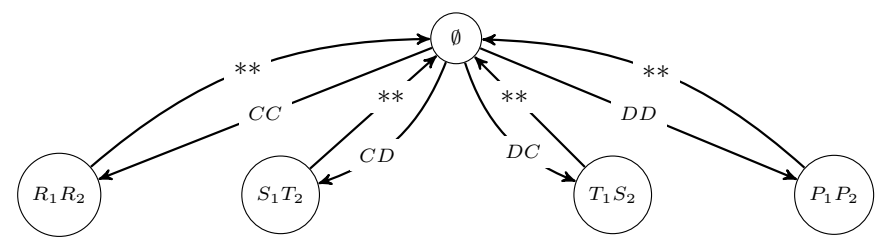

Figure 3: Arena of the Iterated Prisoner's dilemma.

deed, the same Nash equilibria would emerge if, for example, one takes instead of the mean-payoff the maximal payoff that repeats infinitely often.

More generally, given a game in strategic form, and a preference relation $\preceq_{i}$ over its set of possible outcomes $A$, one can define a new preference relation $\preceq_{i}^{\infty}$ over $A^{\omega}$ by assigning a payoff to every subset of $A$ and assigning to each play the set $\inf (\pi) \subseteq A$ of outcomes in $A$ that appear infinitely often in $\pi$. Formally, let $F_{i}: 2^{A} \rightarrow \mathbb{Z}$ be a function mapping subsets of $A$ to integers, and define $\pi \preceq_{i}^{\infty} \pi^{\prime}$ iff $F_{i}(\inf (\pi)) \leq F_{i}\left(\inf \left(\pi^{\prime}\right)\right){ }^{10}$ For example, for the iterated prisoners' dilemma, setting $F_{i}(X)$ to be the number of elements $y$ in $A$ such that $y \prec_{i} x$ where $x$ is a $\preceq_{i}$-maximal element of $X$, results in a game with the same set of Nash equilibria as in the mean payoff version.

We formalise the infinitely repeated prisoner's dilemma as an objective-LTL game. The arena is in Figure 3 . The preferences, for agent $\alpha_{i}$, are defined by the objective $S_{i} \triangleq\left\langle\mathrm{f}_{i}, \varphi_{i}^{1}, \varphi_{i}^{2}, \varphi_{i}^{3}, \varphi_{i}^{4}\right\rangle$ where $\varphi_{i}^{1} \triangleq \mathrm{GF} \mathbf{S}_{i}, \varphi_{i}^{2} \triangleq \mathrm{GF} \mathbf{P}_{i}, \varphi_{i}^{3} \triangleq \mathrm{GF} \mathbf{R}_{i}$, and $\varphi_{i}^{4} \triangleq \mathrm{GF} \mathbf{T}_{i}$, and $\mathrm{f}_{i}$ as before.

\subsection{Illustrating GRADEDSL: uniqueness of solutions}

We illustrate how to express with GRADEDSL some important solution concepts in Game Theory. We start with the concept of winning strategy that is useful in zero-sum games, and then we analyse the well known solution concepts, such as Nash and subgame-perfect equilibria, that are used in non zero-sum games. We use ordinary SL quantifiers (i.e., $\langle\langle x\rangle\rangle,[[x]]$ ) since, as observed in Section 2, these are expressible in GradedSL.

\subsubsection{Winning strategies}

In two-player win-lose zero-sum games the main solution concept is the winning strategy. That is, if $G$ is such an objective-LTL game, then a strategy for agent $\alpha_{1}$ is winning if and only if for all strategies of agent $\alpha_{2}$, the resulting induced play has payoff 1 for agent $i$. This can be expressed in SL as follows:

$$
\phi_{W S}(x) \triangleq\left[[y]\left(\alpha_{1}, x\right)\left(\alpha_{2}, y\right) \bigvee_{f_{1}(a)=1} \eta_{1}^{a}\right.
$$

where $\eta_{1}^{a}$ is the LTL formula defined in Section 4.2. Thus, the following formula expresses that there is a unique winning strategy for player 1 :

$$
\langle\langle x\rangle\rangle^{\geq 1} \phi_{W S}(x) \wedge \neg\langle\langle x\rangle\rangle^{\geq 2} \phi_{W S}(x)
$$

${ }^{10}$ This is reminiscent of the Muller acceptance condition in automata theory. 
Observe that this is a formula of $\operatorname{GRADED}_{\mathbb{N}} \mathrm{SL}[\mathrm{NG}]$ of alternation number 1 . Thus, by Theorem 3.3 we get:

Theorem 4.1. Deciding if a given player in a two-player zero-sum objective-

LTL game has a unique winning strategy can be solved in 2EXPTiME.

We illustrate with an example. In [44 the authors describe a two-player game named "Cop and the Robber", played in a maze, in which the objective of the Robber is to reach an exit (and thus the objective of the Cop is to ensure the Robber never reaches the exit). The authors describe two closely related mazes in which the Robber has, respectively, exactly one and exactly two winning strategies. Both these properties can be easily expressed by GSL. For instance, the Robber has a single LTL objective Fexit, and the following formula of GRADED $\mathbb{N} S L$ expresses that the Robber has exactly one winning strategy:

$$
\left.\langle\langle x\rangle\rangle^{\geq 1} \llbracket y \rrbracket(\text { Robber, } x)(\text { Cop, } y) \text { F exit } \wedge \neg\langle\langle x\rangle\rangle^{\geq 2} \llbracket[y](\text { Robber, } x) \text { (Cop, } y\right) \text { F exit. }
$$

\subsubsection{Nash Equilibria}

The central solution concept in non zero-sum games is the Nash Equilibrium. A tuple of strategies, one for each player, is called a strategy profile. A strategy profile is a Nash equilibrium (NE) if no agent can increase his payoff by unilaterally choosing a different strategy. A game may have zero, one, or many NE.

Consider the case that each agent $\alpha_{i}$ has a general objective tuple $S_{i} \triangleq$ $\left\langle\mathrm{f}_{i}, \varphi_{i}^{1}, \ldots, \varphi_{i}^{m}\right\rangle$. Recall the definition of the LTL formulas $\eta_{i}^{a}$ from Section 4.2

For $\bar{x} \triangleq\left(x_{1} \ldots x_{n}\right)$ and $\bar{y} \triangleq\left(y_{1} \ldots y_{n}\right)$, the following formula says that if all agents follow $\bar{x}$, then no agent $i$ gets a better payoff by deviating and following $y_{i}$ :

$$
\phi_{D E V}(\bar{x}, \bar{y}) \triangleq \bigwedge_{i=1}^{n} \bigwedge_{a \in\{0,1\}^{m}}\left(b\left(\bar{x} / y_{i}\right) \eta_{i}^{a}\right) \rightarrow \bigvee_{f_{i}\left(a^{\prime}\right) \geq f_{i}(a)} b(\bar{x}) \eta_{i}^{a^{\prime}}
$$

where $b(\bar{x})=\left(\alpha_{1}, x_{1}\right) \ldots\left(\alpha_{n}, x_{n}\right)$, and

$$
b\left(\bar{x} / y_{i}\right)=\left(\alpha_{1}, x_{1}\right) \ldots\left(\alpha_{i-1}, x_{i-1}\right)\left(\alpha_{i}, y_{i}\right)\left(\alpha_{i+1}, x_{i+1}\right) \ldots\left(\alpha_{n}, x_{n}\right) .
$$

Then, the following SL formula says that $\bar{x}$ is a NE

$$
\phi_{N E}(\bar{x}) \triangleq \llbracket\left[y_{1} \rrbracket \ldots \llbracket\left[y_{n} \rrbracket\right] \phi_{D E V}(\bar{x}, \bar{y}),\right.
$$

and the following GRADED $\mathrm{N} S[\mathrm{NG}]$ formula expresses that there is a unique $\mathrm{NE}$ :

$$
\left\langle\left\langle x_{1}, \ldots, x_{n}\right\rangle\right\rangle^{\geq 1}\left[\llbracket y_{1}\right] \ldots \llbracket\left[y _ { n } \rrbracket \phi _ { D E V } ( \overline { x } , \overline { y } ) \wedge \neg \langle \langle x _ { 1 } , \ldots , x _ { n } \rangle \rangle ^ { \geq 2 } \left[[ y _ { 1 } ] \ldots \llbracket \left[y_{n} \rrbracket \phi_{D E V}(\bar{x}, \bar{y})\right.\right.\right.
$$

Thus, by Theorem 3.3 we get: 
Theorem 4.2. Deciding if an objective-LTL game has a unique NE can be solved in 2EXPTIME.

Rational synthesis can be formalised as the problem of deciding if a given game has a NE such that the resulting play satisfies a given LTL formula $\Psi$. In our setting, we get the following result by replacing $\phi_{D E V}$ by $\Psi \wedge \phi_{D E V}$ in the previous formula:

Theorem 4.3. Deciding if an objective-LTL game has a unique NE satisfying an LTL formula $\Psi$ can be solved in 2ExPTime.

\subsubsection{Pareto efficiency}

A strategy profile is said to be Pareto efficient (PE) if there is no other strategy profile that makes some agent better off without making another agent worse off. The formula $\phi_{P E}(\bar{x}) \triangleq \llbracket\left[x_{1}^{\prime} \rrbracket \ldots \llbracket \llbracket x_{n}^{\prime} \rrbracket \psi\left(\bar{x}, \overline{x^{\prime}}\right)\right.$ expresses that $\bar{x}$ is $\mathrm{PE}$ where $\psi\left(\bar{x}, \overline{x^{\prime}}\right)$ is

$$
\bigwedge_{i \leq n} \bigwedge_{\left(a, a^{\prime}\right) \in X_{i}}\left(\left(b(\bar{x}) \eta_{i}^{a} \wedge b\left(\overline{x^{\prime}}\right) \eta_{i}^{a^{\prime}}\right) \rightarrow \bigvee_{j \neq i} \bigvee_{\left(c, c^{\prime}\right) \in Y_{i}}\left(b(\bar{x}) \eta_{j}^{c} \wedge b\left(\overline{x^{\prime}}\right) \eta_{j}^{c^{\prime}}\right)\right)
$$

where $\left(a, a^{\prime}\right) \in X_{i}$ iff $\mathrm{f}_{i}\left(a^{\prime}\right)>\mathrm{f}_{i}(a)$, where $\left(c, c^{\prime}\right) \in Y_{i}$ iff $\mathrm{f}_{j}\left(c^{\prime}\right)<\mathrm{f}_{j}(c), b(\bar{x}) \triangleq$ $\left(\alpha_{1}, x_{1}\right) \ldots\left(\alpha_{n}, x_{n}\right)$, and $b\left(\overline{x^{\prime}}\right) \triangleq\left(\alpha_{1}, x_{1}^{\prime}\right) \ldots\left(\alpha_{n}, x_{n}^{\prime}\right)$. Using graded modalities, we can thus express that there is a unique PE using the following GRADED $\mathbb{N} S L[N G]$ formula of alternation number 1 :

$$
\left\langle\left\langle x_{1}, \ldots, x_{n}\right\rangle\right\rangle^{\geq 1} \phi_{P E}(\bar{x}) \wedge \neg\left\langle\left\langle x_{1}, \ldots, x_{n}\right\rangle\right\rangle^{\geq 2} \phi_{P E}(\bar{x}) .
$$

Thus, by using Theorem 3.3 we get:

Theorem 4.4. Deciding if an objective-LTL game has a unique Pareto efficient profile can be solved in 2EXPTime.

\subsubsection{Subgame-perfect equilibria}

Finally, we end with a discussion of the problem of deciding if a game has a unique subgame-perfect equilibrium, and give an upper bound. It has been argued (in [45, 8]) that NE may be implausible when used for sequential games (of which infinitely repeating games are central examples), and that a more robust notion is subgame-perfect equilibrium [46].

Informally, a strategy profile is a subgame-perfect equilibrium if it is a $\mathrm{NE}$ in every reachable subgame. Here is the mathematical definition instantiated for quasi-quantitative games (following the definition in 42 for extensive-form games). Given a history $h \in \operatorname{Hst}\left(s_{I}\right)$ ending in state $s$, say $h=u s$, and a strategy $\sigma \in \operatorname{Str}\left(s_{I}\right)$, the $h$-translation of $\sigma$ is the strategy $\sigma_{\mid h} \in \operatorname{Str}(s)$ that maps $h^{\prime} \in \operatorname{Hst}(s)$ to $\sigma\left(u \cdot h^{\prime}\right)$. Given a quasi-quantitative game $\mathcal{G}=\left\langle\mathcal{A}, S_{1}, \ldots, S_{n}\right\rangle$, 
the profile $\sigma_{1}, \ldots, \sigma_{n}$ is a subgame-perfect equilibrium (SPE) iff for all histories $h \in \operatorname{Hst}\left(s_{I}\right)$, the profile $\sigma_{1 \mid h}, \ldots, \sigma_{n \mid h}$ is a NE in $\mathcal{G}=\left\langle\mathcal{A}_{\mid h}, S_{1 \mid h}, \ldots, S_{n \mid h}\right\rangle$ where $\mathcal{A}_{\mid h}$ is the same arena as $\mathcal{A}$ but with $s$ as the initial state, and if $S=$ $\left\langle\mathrm{f}_{i}, L_{i}^{1}, \ldots, L_{i}^{m}\right\rangle$ then $S_{\mid h}=\left\langle\mathrm{f}_{i}, H_{i}^{1}, \ldots, H_{i}^{m}\right\rangle$ where $\pi \in H_{i}^{j}$ iff $u \cdot \pi \in L_{i}^{j}$. The point is that the payoff in $\mathcal{G}_{\mid h}$ applies to the whole path (i.e., starting from $s_{I}$ ), even though the strategies only apply after $h$.

Using the notation in the previous paragraph, suppose each $L_{i}^{j}$ is prefixindependent, i.e., $\pi \in L_{i}^{j}$ iff $\pi_{\geq n} \in L_{i}^{j}$ for all $n \geq 1$ (here $\pi_{\geq n}$ is the suffix of $\pi$ starting at position $n$ ). In this case, $H_{i}^{j}=L_{i}^{j}$. Observe that the assumption that the objectives are prefix-independent is not too restrictive. Indeed, as discussed in Section 2.9, in many infinitely repeated games the outcome ignores all finite prefixes of the play.

Thus, suppose $\mathcal{G}$ is an objective-LTL game in which the set of models of each $\varphi_{i}^{j}$ is prefix-independent. The following formula of SL expresses that $\bar{x}$ is an SPE 11

$$
\phi_{S}(\bar{x}) \triangleq \llbracket\left[z_{1} \rrbracket \ldots \llbracket\left[z_{n}\right] \rrbracket\left[y_{1}\right] \ldots \llbracket\left[y_{n}\right]\left(\alpha_{1}, z_{1}\right) \ldots\left(\alpha_{n}, z_{n}\right) \mathrm{G}_{\phi_{D E V}}(\bar{x}, \bar{y}) .\right.
$$

Indeed, since $[\cdot]]$ commutes with $\mathrm{G}$, the formula $\phi_{S}(\bar{x})$ is equivalent to

$$
\llbracket\left[z_{1}\right] \ldots \llbracket\left[z_{n} \rrbracket\left(\alpha_{1}, z_{1}\right) \ldots\left(\alpha_{n}, z_{n}\right) \mathrm{G} \phi_{N E}(\bar{x})\right.
$$

which is true in $\mathcal{A}, \chi, s_{I}$ iff for all histories $h$ starting in $s_{I}$ and ending, say, in state $s$, we have that $\mathcal{A}, \chi^{\prime}, s \models \phi_{N E}$ where the strategy $\chi^{\prime}(x)$ is the $h$-translation of the strategy $\chi(x)$, i.e., the profile $\chi^{\prime}\left(x_{1}\right), \ldots, \chi^{\prime}\left(x_{n}\right)$ is a NE in $\mathcal{G}_{\mid h}$.

Using graded modalities, we can thus express there is a unique SPE (assuming each $\varphi_{i}^{j}$ is prefix-independent) as the following GRADED $\mathbb{N} S L[N G]$ formula of alternation number 1 :

$$
\left\langle\left\langle x_{1}, \ldots, x_{n}\right\rangle\right\rangle^{\geq 1} \phi_{S}(\bar{x}) \wedge \neg\left\langle\left\langle x_{1}, \ldots, x_{n}\right\rangle\right\rangle^{\geq 2} \phi_{S}(\bar{x}) .
$$

Thus, by using Theorem 3.3 we get:

Theorem 4.5. Deciding if an objective-LTL game with prefix-independent objectives $\varphi_{i}^{j}$ has a unique SPE can be solved in 2EXPTime.

\section{Conclusion}

The Nash equilibrium is the foundational solution concept in game theory. The last twenty years have witnessed the introduction of many logical formalisms for modeling and reasoning about solution concepts, and NE in particular [3, 4, 6, 47, 48, 9, 7]. These formalisms are useful for addressing qualitative questions

\footnotetext{
${ }^{11}$ Previous formalisations of SPE overlook the need for a condition like prefixindependence $45,4,8$.
} 
such as "does the game admit a Nash equilibrium?". Among others, Strategy Logic (SL) has come to the fore as a general formalism that can express and solve this question, for LTL objectives, in 2ExpTime. Contrast this with the fact that this question is 2ExPTimE-complete even for two player zero-sum LTL games [4].

One of the most important questions about NE in computational game theory is "does the game admit more than one NE?" [14, 15] This issue has been deeply investigated in game theory and is shown to be very challenging [16, 17, 18, 13, 19, 50, 51, 52. Prior to this work, no logic-based technique, as far as we know, solved the corresponding decision problem, i.e., whether or not a given game has a unique NE ${ }^{12}$ In this paper we introduced GRADEDSL to address and solve the unique NE problem. We have demonstrated that GRADEDSL is elegant, simple, and very powerful, and can solve the unique NE problem for LTL objectives in 2EXPTIME, and thus at the same complexity that is required to merely decide if a $\mathrm{NE}$ exists. We also illustrated that one can express the uniqueness of other solution concepts, including winning strategies, subgame-perfect equilibria, and Pareto-efficient profiles, all in 2ExPTimE. We remark that the exact complexity of the existence of a unique NE, and of counting the number of NE, has been studied for other representations of games, notably games in strategic form with rational co-efficients and considering mixed strategies [54, 55]. The exact complexity of the existence of NE in our representation is currently unknown. Finally, our work gives the first algorithmic solution to the model-checking problem of a graded variant of $\mathrm{ATL}^{\star}$, and proves it to be 2EXPTIME-COMPLETE.

In the multi-agent setting, reasoning about epistemic alternatives plays a key role. Thus, an important extension would be to combine the knowledge operators in SLK [56] with the graded quantifiers we introduced for GRADEDSL. Since strategic reasoning under imperfect information has an undecidable modelchecking problem [57, one may restrict to memoryless strategies as was done for SLK. More involved, would be to add grades to the knowledge operators, thus being able to express "there exists at least $g$ equivalent worlds" 58.

Finally, another direction is to implement GRADEDSL and its model-checking procedure in a formal verification tool. A reasonable approach would be, for example, to extend the tool SLK-MCMAS [56, 59].

\section{Acknowledgments}

We thank Michael Wooldridge for pointing out the importance of uniqueness of Nash Equilibria in game theory. Benjamin Aminof is supported by the Austrian National Research Network S11403-N23 (RiSE) of the Austrian Science Fund (FWF) and by the Vienna Science and Technology Fund (WWTF)

\footnotetext{
${ }^{12}$ In the related work section we discussed the logic GSL [31, 53] that, although motivated by the need to address the unique NE problem, only supplies a model-checking algorithm for a very small fragment of GSL that, cannot express LTL goals, it is assumed, is not able to express the existence of $\mathrm{NE}$.
} 
through grant ICT12-059. Sasha Rubin is supported by a Marie Curie fellowship of the Istituto Nazionale di Alta Matematica. Aniello Murano is partially supported by the GNCS 2016 project: Logica, Automi e Giochi per Sistemi Auto-adattivi.

\section{References}

[1] B. Aminof, V. Malvone, A. Murano, S. Rubin, Graded strategy logic: Reasoning about uniqueness of nash equilibria, in: AAMAS 2016, IFAAMAS, 2016, pp. 698-706.

[2] B. Aminof, V. Malvone, A. Murano, S. Rubin, Extended graded modalities in strategy logic, in: Proceedings of the 4th International Workshop on Strategic Reasoning, SR 2016, New York City, USA, 10th July 2016., 2016, pp. 1-14. doi:10.4204/EPTCS.218.1.

[3] F. Mogavero, A. Murano, M. Vardi, Reasoning About Strategies., in: FSTTCS'10, LIPIcs 8, Leibniz-Zentrum fuer Informatik, 2010, pp. 133144. doi:10.4230/LIPICs.FSTTCS.2010.133.

[4] F. Mogavero, A. Murano, G. Perelli, M. Vardi, Reasoning About Strategies: On the Model-Checking Problem., TOCL 15 (4) (2014) 34:1-42. doi: 10.1145/2631917.

[5] R. Alur, T. Henzinger, O. Kupferman, Alternating-Time Temporal Logic., JACM 49 (5) (2002) 672-713. doi:10.1145/585265.585270.

[6] K. Chatterjee, T. Henzinger, N. Piterman, Strategy Logic., IC 208 (6) (2010) 677-693. doi:10.1016/j.ic.2009.07.004.

[7] J. Gutierrez, P. Harrenstein, M. Wooldridge, Reasoning about equilibria in game-like concurrent systems, in: KR 2014, AAAI, 2014.

[8] O. Kupferman, G. Perelli, M. Y. Vardi, Synthesis with rational environments, in: EUMAS 2014, 2014, pp. 219-235. doi:10.1007/ 978-3-319-17130-2\_15.

[9] F. Belardinelli, A logic of knowledge and strategies with imperfect information, in: LAMAS 15, 2015.

[10] F. Belardinelli, A. Lomuscio, A. Murano, S. Rubin, Verification of broadcasting multi-agent systems against an epistemic strategy logic, in: Proceedings of the Twenty-Sixth International Joint Conference on Artificial Intelligence, IJCAI 2017, Melbourne, Australia, August 19-25, 2017, 2017, pp. 91-97. doi:10.24963/ijcai.2017/14.

[11] J. Gutierrez, A. Murano, G. Perelli, S. Rubin, M. Wooldridge, Nash equilibria in concurrent games with lexicographic preferences, in: Proceedings 
of the Twenty-Sixth International Joint Conference on Artificial Intelligence, IJCAI 2017, Melbourne, Australia, August 19-25, 2017, 2017, pp. 1067-1073. doi:10.24963/ijcai.2017/148

[12] R. Berthon, B. Maubert, A. Murano, S. Rubin, M. Y. Vardi, Strategy logic with imperfect information, in: 32nd Annual ACM/IEEE Symposium on Logic in Computer Science, LICS 2017, Reykjavik, Iceland, June 20-23, 2017, 2017, pp. 1-12. doi:10.1109/LICS.2017.8005136.

[13] E. Altman, H. Kameda, Y. Hosokawa, Nash equilibria in load balancing in distributed computer systems, IGTR 4 (2) (2002) 91-100. doi:10.1142/ S0219198902000574.

[14] G. Papavassilopoulos, J. B. Cruz, On the uniqueness of nash strategies for a class of analytic differential games, Journal of Optimization Theory and Applications 27 (2) (1979) 309-314. doi:10.1007/BF00933234.

[15] R. Cornes, R. Hartley, T. Sandler, An elementary proof via contraction, Journal of Public Economic Theory 1 (4) (1999) 499-509. doi:10.1111/ 1097-3923.00023.

[16] J. B. D. Simchi-Levi, X. Chen, The Logic of Logistics: Theory, Algorithms, and Applications for Logistics Management, Science and Business Media, Springer, 2013.

[17] Y. Zhang, M. Guizani, Game Theory for Wireless Communications and Networking, CRC Press, 2011.

[18] L. Pavel, Game Theory for Control of Optical Networks, Science and Business Media, Springer, 2012. doi:10.1007/978-0-8176-8322-1.

[19] A. Orda, R. Rom, N. Shimkin, Competitive routing in multiuser communication networks, IEEE/ACM Trans. Netw. 1 (5) (1993) 510-521. doi:10.1109/90.251910.

[20] K. Fine, In So Many Possible Worlds., NDJFL 13 (1972) 516-520.

[21] E. Grädel, M. Otto, E. Rosen, Two-Variable Logic with Counting is Decidable., in: LICS'97, IEEE Computer Society, 1997, pp. 306-317.

[22] B. Hollunder, F. Baader, Qualifying Number Restrictions in Concept Languages., in: KR'91, Kaufmann, 1991, pp. 335-346.

[23] O. Kupferman, U. Sattler, M. Vardi, The Complexity of the Graded muCalculus., in: CADE'02, LNCS 2392, Springer, 2002, pp. 423-437. doi:10.1007/3-540-45620-1\_34.

[24] A. Bianco, F. Mogavero, A. Murano, Graded Computation Tree Logic., TOCL 13 (3) (2012) 25:1-53. doi:10.1145/2287718.2287725. 
[25] S. Rubin, B. Aminof, A. Murano, On CTL* with graded path modalities, in: LPAR 20, 2015.

[26] O. Kupferman, M. Vardi, P. Wolper, Module Checking., IC 164 (2) (2001) $322-344$.

[27] W. Jamroga, A. Murano, On Module Checking and Strategies., in: AAMAS'14, IFAAMAS, 2014, pp. 701-708.

[28] B. Aminof, A. Legay, A. Murano, O. Serre, $\mu$-calculus pushdown module checking with imperfect state information, in: IFIP-TCS'08, Vol. 273 of IFIP, Springer, 2008, pp. 333-348.

[29] A. Ferrante, A. Murano, M. Parente, Enriched Mu-Calculi Module Checking., LMCS 4 (3) (2008) 1-21. doi:10.2168/LMCS-4(3:1) 2008.

[30] M. Faella, M. Napoli, M. Parente, Graded Alternating-Time Temporal Logic., FI 105 (1-2) (2010) 189-210.

[31] V. Malvone, F. Mogavero, A. Murano, L. Sorrentino, On the counting of strategies, in: TIME 2015, 2015, pp. 170-179. doi:10.1109/TIME.2015. 19.

[32] F. Mogavero, A. Murano, G. Perelli, M. Vardi, What Makes ATL* Decidable? A Decidable Fragment of Strategy Logic., in: CONCUR'12, LNCS 7454, Springer, 2012, pp. 193-208. doi:10.1007/978-3-642-32940-1 $-15$.

[33] J. Gutierrez, P. Harrenstein, M. Wooldridge, Expresiveness and complexity results for strategic reasoning, in: LIPIcs, Vol. 42, 2015.

[34] F. Mogavero, A. Murano, M. Vardi, Relentful Strategic Reasoning in Alternating-Time Temporal Logic., in: LPAR'10, LNAI 6355, Springer, 2010, pp. 371-387.

[35] K. Leyton-Brown, Y. Shoham, Essentials of Game Theory: A Concise, Multidisciplinary Introduction (Synthesis Lectures on Artificial Intelligence and Machine Learning), M\&C, 2008. doi:10.2200/ S00108ED1V01Y200802AIM003.

[36] F. Mogavero, Logics in Computer Science., Ph.D. thesis, Universitá degli Studi di Napoli Federico II, Napoli, Italy (January 2011).

[37] O. Kupferman, M. Vardi, P. Wolper, An Automata Theoretic Approach to Branching-Time Model Checking., JACM 47 (2) (2000) 312-360. doi: 10.1145/333979.333987.

[38] W. Thomas, Automata on infinite objects, Handbook of theoretical computer science, Volume B (1990) 133-191. 
[39] E. A. Emerson, C. S. Jutla, Tree automata, mu-calculus and determinacy (extended abstract), in: ASFCS 1991, 1991, pp. 368-377. doi:10.1109/ SFCS.1991.185392.

[40] V. Bárány, L. Kaiser, A. M. Rabinovich, Expressing cardinality quantifiers in monadic second-order logic over trees, Fundam. Inform. 100 (1-4) (2010) 1-17. doi:10.3233/FI-2010-260.

[41] D. E. Muller, P. E. Schupp, Simulating alternating tree automata by nondeterministic automata: New results and new proofs of the theorems of rabin, mcnaughton and safra, Theor. Comput. Sci. 141 (1\&2) (1995) 69107. doi:10.1016/0304-3975(94)00214-4.

[42] M. Osborne, A. Rubinstein, A course in game theory, MIT press, 1994.

[43] K. G. Binmore, Fun and Games: A Text on Game Theory, D.C. Heath, 1992.

[44] V. Malvone, A. Murano, L. Sorrentino, Games with additional winning strategies, in: CILC, 2015, CEUR, 2015, pp. 175-180.

[45] M. Ummels, Rational behaviour and strategy construction in infinite multiplayer games, in: FSTTCS, 2006, pp. 212-223. doi:10.1007/11944836 -21.

[46] R. Selten, Spieltheoretische behandlung eines oligopolmodells mit nachfragetragheit., Zeitschrift fur die gesamte Staatswissenschaft 121 (1965) 301-324.

[47] T. Brihaye, A. D. C. Lopes, F. Laroussinie, N. Markey, ATL with strategy contexts and bounded memory, in: LFCS 2009, 2009, pp. 92-106. doi: 10.1007/978-3-540-92687-0\_7.

[48] A. D. C. Lopes, F. Laroussinie, N. Markey, ATL with strategy contexts: Expressiveness and model checking, in: FSTTCS 2010, 2010, pp. 120-132. doi:10.4230/LIPICs.FSTTCS.2010.120.

[49] A. Pnueli, R. Rosner, On the Synthesis of a Reactive Module., in: POPL'89, Association for Computing Machinery, 1989, pp. 179-190.

[50] H. R. V. T. C. Bergstrom, L. E. Blume, On the private provision of public goods, Journal of Public Economics 29 (1) (1986) 25-49.

[51] C. D. Fraser, The uniqueness of nash equilibrium in the private provision of public goods: an alternative proof., Journal of Public Economics 49 (3) (1992) 389-390.

[52] A. Glazer, K. A. Konrad, Private provision of public goods, limited tax deducibility, and crowding out, FinanzArchiv / Public Finance Analysis 50 (2) (1993) 203-216. 
[53] V. Malvone, F. Mogavero, A. Murano, L. Sorrentino, Reasoning about graded strategy quantifiers, Inf. Comput. (to appear).

[54] I. Gilboa, E. Zemel, Nash and correlated equilibria: Some complexity considerations, Games and Economic Behavior 1 (1) (1989) 80-93.

[55] V. Conitzer, T. Sandholm, New complexity results about nash equilibria, Games and Economic Behavior 63 (2) (2008) 621-641. doi:10.1016/j. geb.2008.02.015.

[56] P. Čermák, A. Lomuscio, F. Mogavero, A. Murano, MCMAS-SLK: A Model Checker for the Verification of Strategy Logic Specifications., in: CAV'14, LNCS 8559, Springer, 2014, pp. 524-531. doi:10.1007/ 978-3-319-08867-9\_34.

[57] C. Dima, F. Tiplea, Model-checking ATL under Imperfect Information and Perfect Recall Semantics is Undecidable., Tech. rep., arXiv (2011).

[58] W. van der Hoek, J. Meyer, Graded modalities in epistemic logic, in: LFCS'92, Vol. 620, Springer, 1992, pp. 503-514.

[59] P. Cermák, A. Lomuscio, A. Murano, Verifying and synthesising multiagent systems against one-goal strategy logic specifications, in: AAAI 2015, 2015, pp. 2038-2044. 